\title{
Calculation Experiment Technology
}

\author{
Vladilen F. Minin, Igor V. Minin and Oleg V. Minin \\ Novosibirsk State Technical University \\ Russia
}

\section{Introduction}

There are two common approaches for numerical solution of continuum equations in mechanics: Lagrangian and Eulerian. The choice usually depends on exploiting specific features of these approaches that are suitable for the problem at hand. In the Lagrangian approach the computational grid that discretizes the domain deforms with the material. However Lagrangian method is not suitable for applications involving large distortion and large rotation, or for cases where boundary itself is modified as the solution proceeds. On the other hand, in the Eulerian approach the computational grid is fixed in space. The material moves through this grid as it flows and deforms. Even though large distortions are handled easily in this method, interface tracking and contact surface algorithms pose considerable difficulty.

Novel methods have been developed that discretize the continuum domain by discrete Lagrangian particles. Harlow's Particle In Cell (PIC) method [1] may be considered to be one of the precursors. This method eliminates the shortcomings of the traditional Lagrangian and Eulerian methods while retaining the good aspects of them. This method allows one to solve a broader class of problems by allowing large distortions and efficient calculation at interfaces. The PIC method also allows precise distinction of material boundaries. In spite of these advantages the PIC method shows certain limitations for problems where variables are history dependent, for example in elasto-plasticity, viscoelasticity, various relaxation processes, and for problems dealing with low pressures. Another shortcoming of this approach is that the solution fluctuates due to the method of discretization of mass, energy and momentum, and the way by which density is calculated. A more serious limitation arises out of the complexity of pressure calculation in a mixed cell.

From the above mentioned of existing computational methods for non-stationary continua it is clear that none of them satisfy the requirements for large-scale computation. The grid and particle methods such as PIC and GAP [2] seem to possess the best characteristics in this regard. Hence these methods were taken up as a basis for developing a new method the method of individual particles (1979, developed under the scientific leaderships of Prof. V.F.Minin) to extend the areas of applicability of the particle methods. Some particle methods in Astrophysical Fluid Dynamics are discussed and available at [3].

On the calculation experiment technology Prof. V.F.Minin is the winner of the State premium of the USSR. 


\section{The general scheme of "Individual Particles" (IP) method}

In the IP (Individual Particles) method, which is described below, a continuum is discretized into small volumes each of which is represented by a particle. All the physical quantities such as mass, velocity, and thermodynamic variables like density, energy etc. of the entire flow field are represented by those of the particles whose coordinates are known at all times. The particles can have changing shapes (as opposed to being point masses) depending on local flow parameters. The particles can also unite or divide into new particles depending on the flow parameters.

The main goal of developing the new method is to eliminate the limitations of both PIC and GAP methods, e.g. oscillation of solutions, complexity of "mixed cell" calculations, nonphysical discontinuities in otherwise continuous solutions etc. However, it is important to preserve basic advantages of the earlier methods such as the natural way of computations for multi-component media experiencing large strain, and transport of materials across interfaces.

Let's write out the set of equations for a two-dimensional plane symmetry or axisymmetric compressible medium in Eulerian coordinates:

$$
\begin{aligned}
& \frac{\partial \mathrm{p}}{\partial \mathrm{t}}+\operatorname{pdiv} \overrightarrow{\mathrm{V}}=0, \\
& \rho \frac{\mathrm{du}}{\mathrm{dt}}=\frac{\partial \mathrm{P}^{\mathrm{zz}}}{\partial \mathrm{z}}+\frac{\partial \mathrm{P}^{\mathrm{zr}}}{\partial \mathrm{r}}+\left(\frac{\mathrm{P}^{\mathrm{zr}}}{\mathrm{r}}\right)^{*}, \\
& \rho \frac{\mathrm{dV}}{\mathrm{dt}}=\frac{\partial \mathrm{P}^{\mathrm{rz}}}{\partial \mathrm{z}}+\frac{\partial \mathrm{P}^{\mathrm{rr}}}{\partial \mathrm{r}}+\left(\frac{\mathrm{P}^{\mathrm{rr}}-\mathrm{P}^{\varphi \varphi}}{\mathrm{r}}\right)^{*}, \\
& \rho \frac{\mathrm{de}}{\mathrm{dt}}=\mathrm{P}^{\mathrm{zz}} \frac{\partial \mathrm{U}}{\partial \mathrm{z}}+\mathrm{P}^{\mathrm{zr}}\left(\frac{\partial \mathrm{V}}{\partial \mathrm{z}}+\frac{\partial \mathrm{U}}{\partial \mathrm{r}}\right)+\mathrm{P}^{\mathrm{rr}} \frac{\partial \mathrm{V}}{\partial \mathrm{r}}+\left(\mathrm{P}^{\varphi \varphi} \frac{\mathrm{V}}{\mathrm{r}}\right), \\
& \mathrm{P}^{\mathrm{zz}}=-\mathrm{p}+\mathrm{S}^{\mathrm{zz}} \text {, } \\
& \mathrm{P}^{\mathrm{rr}}=-\mathrm{p}+\mathrm{S}^{\mathrm{rr}}, \\
& \mathrm{P}^{\varphi \varphi}=-\mathrm{p}-\left(\mathrm{S}^{\mathrm{zz}}+\mathrm{S}^{\mathrm{rr}}\right) \text {, } \\
& \mathrm{P}^{\mathrm{zr}}=\mathrm{S}^{\mathrm{zr}} \text {, } \\
& p=p(\rho, e), \\
& \frac{\mathrm{dS}^{\mathrm{zz}}}{\mathrm{dt}}=2 \mu\left[\frac{\partial \mathrm{U}}{\partial \mathrm{z}}-\frac{1}{3} \operatorname{div} \overline{\mathrm{V}}\right]+\delta^{\mathrm{zz}}, \\
& \frac{\mathrm{d} S^{\mathrm{rr}}}{\mathrm{dt}}=2 \mu\left[\frac{\partial \mathrm{V}}{\partial \mathrm{r}}-\frac{1}{3} \operatorname{div} \overline{\mathrm{V}}\right]+\delta^{\mathrm{rr}}, \\
& \frac{\mathrm{dS}^{\mathrm{zr}}}{\mathrm{dt}}=\mu\left[\frac{\partial \mathrm{U}}{\partial \mathrm{r}}+\frac{\partial \mathrm{V}}{\partial \mathrm{z}}\right]+\delta^{\mathrm{zr}}, \\
& \operatorname{div} \overline{\mathrm{V}}=\frac{\partial \mathrm{U}}{\partial \mathrm{z}}+\frac{\partial \mathrm{V}}{\partial \mathrm{r}}+\left(\frac{\mathrm{V}}{\mathrm{r}}\right)^{*}, \\
& \delta^{\mathrm{zZ}}=\mathrm{S}^{\mathrm{zr}}\left(\frac{\partial \mathrm{V}}{\partial \mathrm{z}}-\frac{\partial \mathrm{U}}{\partial \mathrm{r}}\right),
\end{aligned}
$$




$$
\begin{gathered}
\delta^{\mathrm{rr}}=\mathrm{S}^{\mathrm{zr}}\left(\frac{\partial \mathrm{U}}{\partial \mathrm{r}}-\frac{\partial \mathrm{V}}{\partial \mathrm{z}}\right), \\
\delta^{\mathrm{zr}}=\frac{\mathrm{S}^{\mathrm{rr}}-\mathrm{S}^{\mathrm{zz}}}{2}\left(\frac{\partial \mathrm{V}}{\partial \mathrm{z}}-\frac{\partial \mathrm{U}}{\partial \mathrm{r}}\right), \\
\left(\mathrm{S}^{\mathrm{zz}}\right)^{2}+\left(\mathrm{S}^{\mathrm{rr}}\right)^{2}+\left(\mathrm{S}^{\mathrm{zr}}\right)^{2}+\mathrm{S}^{\mathrm{zz}} \mathrm{S}^{\mathrm{rr}} \leq \frac{1}{3} \sigma_{0}^{2}
\end{gathered}
$$

Here the variables are: $\mathrm{t}$ - time, $\mathrm{r}, \mathrm{z}$ - spatial coordinates ( $\mathrm{z}$ - axes of a symmetry in cylindrical coordinates), $\rho$ - density, $U$ - velocity in $z$-direction, $V$ - velocity in r-direction, $\mathrm{P}^{\mathrm{ij}}$ _ components of the stress tensor, $S^{\mathrm{ij}}$ - deviatoric stress components, $\mathrm{p}$ - hydrostatic pressure, $\mu$ - shear modulus. For the two-dimensional plane symmetry case, the terms marked with an asterisk are set equal to zero.

The equations (2.10-2.12) are obtained from the generalized Hooke's law for an isotropic elastic medium in terms of strain increments and the ensuing stress increments. The magnitudes $\delta^{\mathrm{ij}}$ in the equations (2.14-2.16) represent Yaumann stress corrections due to rotation.

The inequality (2.17) represents von Mises yield condition with yield stress of $\sigma_{0}$. In the plastic range the inequality in (2.17) is enforced by multiplying

$$
\frac{\sqrt{\frac{1}{3}} \sigma_{0}}{\left(\mathrm{~S}^{\mathrm{zZ}}\right)^{2}+\left(\mathrm{S}^{\mathrm{rr}}\right)^{2}+\left(\mathrm{S}^{\mathrm{zr}}\right)^{2}+\mathrm{S}^{\mathrm{zz}} \mathrm{S}^{\mathrm{rr}}}
$$

to the deviatoric stress components. In case of inviscid and adiabatic flow of fluids the set of equations (2.1-2.17) become the equations of gas dynamics:

$$
\begin{gathered}
\frac{\mathrm{d} \rho}{\mathrm{dt}}+\rho\left[\frac{\partial \mathrm{U}}{\partial \mathrm{z}}+\frac{\partial \mathrm{V}}{\partial \mathrm{r}}+\left(\frac{\mathrm{V}}{\mathrm{r}}\right)^{*}\right]=0, \\
\rho \frac{\mathrm{dU}}{\mathrm{dt}}+\frac{\partial \mathrm{p}}{\partial \mathrm{z}}=0 \\
\rho \frac{\mathrm{dV}}{\mathrm{dt}}+\frac{\partial \mathrm{p}}{\partial \mathrm{r}}=0 \\
\frac{\mathrm{de}}{\mathrm{dt}}-\mathrm{p} \frac{1}{\rho^{2}} \frac{\mathrm{dp}}{\mathrm{dt}}=0
\end{gathered}
$$

and the equation of state,

$$
p=p(\rho, e),
$$

For the two-dimensional plane symmetry case, the terms marked with an asterisk in equations (2.1-2.22) are set equal to zero. The set of equations (2.1-2.17) and (2.18-2.22) containing spatial partial derivatives of stress tensor, pressure and velocity components apply to discrete Lagrangian volumes. As a result, the only additional condition that need to be enforced for cases where more than one material are involved, is the continuity of the normal stress and normal component of velocity at the boundaries.

The basic idea of this method is the following. The set of equations (2.1-2.17) or (2.18-2.22), describing the motion is numerically integrated for each particle. Two grids are considered for the calculation purpose. A Lagrangian grid on which all flow parameters are defined, and aEulerian grid, which is arbitrarily defined at each time step. The nodes in the Lagrangian grid coincide with the particles. Pressure is calculated from the internal energy and density. Then pressure, stress tensor and the velocity fields are mapped to the Eulerian 
grid on which all necessary derivatives are carried out. Then the values of these derivatives are mapped back on to the Lagrangian grid by interpolation. The application of conservation laws (energy, mass and momentum) in finite difference form results in the new values of density, velocity and internal energy at the nodes (centers of particles) of the Lagrangian grid. New positions of the particles are then determined from the new velocity field. If required, uniformity of particle distribution can be achieved by merging smaller particles, and dividing larger particles in to smaller ones. This completes the calculation for the given time step. The mapping schemes that map data to and from the Eulerian grid and the Lagrangian grid are similar.

Some of the distinct features of this method are as follows.

1. The problem associated with calculation of pressure in "mixed cells" is removed, since the calculations are performed on the Lagrangian grid comprising of homogeneous material.

2. The density calculation is done on the particles based on the continuity equation. It completely eliminates the fluctuations of solutions that is normally seen in Eulerian calculations.

3. Since the density and other flow parameters change continuously, the condition that limits the minimum number of particle in a cell is the continuity condition itself. It means, that the calculation can be carried out with only one particle in a mesh. This reduces memory size requirement and the computational time by an order of magnitude compared to other particle methods.

4. The information about the particles is necessary only for calculation of the next time step, since after an interpolation of values of derivatives from the Eulerian grid to the particles, the Eulerian grid information is not necessary. This property of the algorithm allows us to reduce computer memory required to store grid information. Calculation is carried out on a minimum necessary "quantum" Eulerian grid, and then moved to the field of particles.

5. One virtue of representing a medium by Lagrangian particles (individual volumes that carry full state information of the flow field at a given point) is the ease of tracking history dependent variables. Any number of history variables can be tracked for a particle. Therefore different bodies can be described by different equations of state. This does not complicate the computational algorithm. For example, to describe an elasticplastic process the additional parameters are the components of stress deviator. Similarly, for calculation of detonation of explosives (HE) (taking into account the kinetics of its decomposition in shock waves) the additional parameters are the values of relative specific volume of solid and gaseous phases and the degree of transformation of HE in the detonation products. The simulation of damage processes, calculation of polymorphic phase changes can be effected similarly. In all such cases only the subroutines that calculate appropriate equation of state, kinetics and rheological conditions are changed. The main program is not affected.

Also, it is easy in IP method to calculate rigid body interaction with deforming medium by not allowing particles to move into the rigid body but allowing them to transfer momentum at the same time.

6. The algorithm of IP method is simple and is homogeneous, that allows one to effectively implement it in parallel and multiprocessor computers.

The various implementations of the IP method differ from each other on the type of Eulerian grid used (regular, irregular, adapted to singularities of flow etc.), mode of division and 
merger of particles, interpolation schemes used to map values from Lagrangian grid to Eulerian grid and back etc. The specific implementation also depends on the computer architecture. In the following pages we describe the implementation in a single processor type computer.

\section{Implementation of the IP method}

In the following, we describe the computational method for non-stationary, inviscid and adiabatic flow in an axi-symmetric or planar symmetric continuum. A computational grid containing discrete particles is overlaid on the physical domain as shown in fig-3.1.

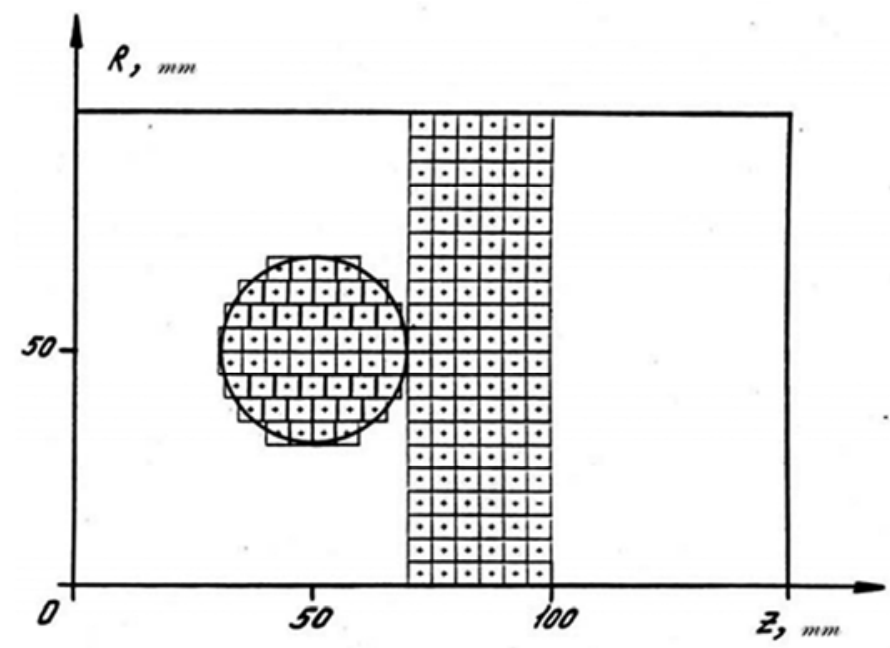

Fig. 3.1. Representation of a domain as particles and individual volumes

Each particle represents a discrete homogeneous volume (that is, the volume occupied by one material) and is characterized by the following parameters: mass, M; coordinates of the center, zand $\mathrm{r}$; components of velocity, $\mathrm{U}$ and $\mathrm{V}$, in $\mathrm{z}$ and $\mathrm{r}$ directions respectively; density, $\rho$; specific internal energy, e; artificialviscosity, q; and a number, $\mathrm{N}_{\mathrm{B}}$ describing its material properties. For solids (media with strength effects), the list of parameters for each particle also includes the components of stress deviator $S^{z z}, S^{r r}, S^{z r}$. Let's assume that in the $z-r$ planethe form of cell is quadrilateral, at the center of which all its parameters are defined. The particles in each cell in the planar case have identical mass, where as in theaxisymmetrical case the mass is proportional to the radial distance of the particles from the axis of symmetry. The boundaries can be rigid, reflective, or boundaries through which substances can enter or leave. In case of axi-symmetric flow, the boundary $r=0$ is the axis of symmetry. We may also include a rigid body, stationary or moving, inside the computational grid. For each particle, the set of equations of gas dynamics (2.18-2.22) is integrated. Unknown values of space derivatives, $\frac{\partial \mathrm{p}}{\partial \mathrm{z}}, \frac{\partial \mathrm{p}}{\partial \mathrm{r}}, \frac{\partial \mathrm{U}}{\partial \mathrm{z}}, \frac{\partial \mathrm{V}}{\partial \mathrm{r}}$ for each particle are determined in the fixed Eulerian grid. The mesh density is chosen so that there is no discontinuity. In most cases this condition is fulfilled by arranging the grid in such a way that each cell contains at least one particle. Further, we shall use a uniform Eulerian grid with square cells. 
Stage-I - interpolation from "particle to grid": The pressure is calculated for each particle using the formula $\tilde{p}=\mathrm{p}+\mathrm{q}$. Here $\mathrm{p}$ is the hydrostatic pressure obtained from equation (2.22), $q$ is the linear artificial viscosity calculated on the previous time step. At the centers of particles, pressurep̃and velocity components $U, V$ are known. These are then interpolated to the nodes of Eulerian grid. The interpolation can be done by various methods, for example, "weighing" on squares denoted by $\mathrm{S}_{\mathrm{N}}^{\mathrm{ij}}$ (defined in Fig.3.2):

$$
A=\frac{\sum_{N} A_{N} S_{N}^{i j}}{\sum_{N} S_{N}^{i j}}
$$

where the indices $I, j$ refer to the node $(I, j)$ of the Eulerian grid, index $N$ refers to the $N^{\text {th }}$ particle, $A=(\tilde{p}, U, V)$. Each particle contributes to the four adjacent nodes. In fig. (3.2) crosses and circles represent centers of two types of particles.The dotted line shows the partition of a cell into squares in inverse proportion to the contribution of each particle to the respective nodes. Accurate interpolation can be produced in many other ways as well.

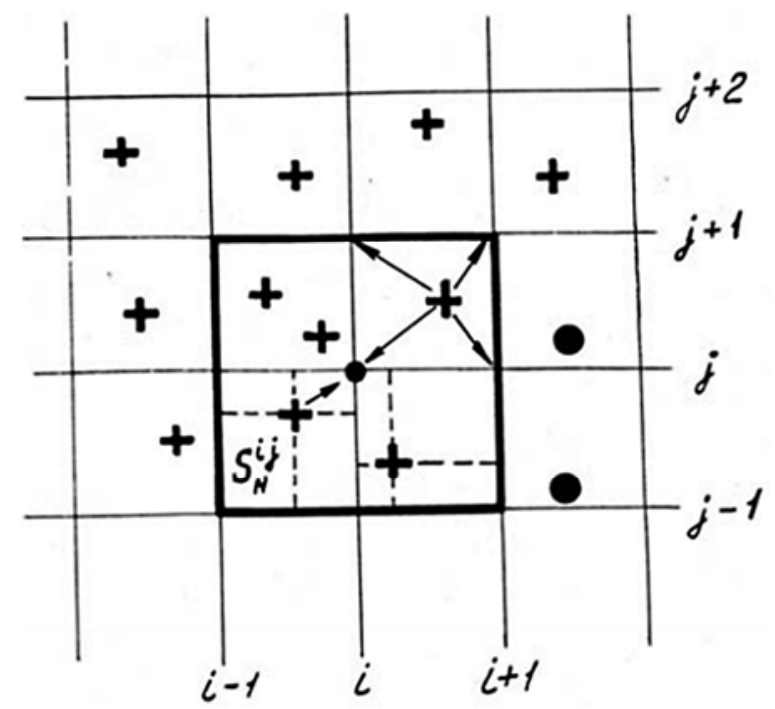

Fig. 3.2. An interpolation of "particle - grid"

In addition to the interpolated values from "particle to grid", each particle also contributes to the information about its membership of the material category to the node. Thus each node can be categorized into the following four classes (fig.3.3):

1. A "Vacuum" node - there are no particles in the adjacent cells.

2. An interior node - the particles in the adjacent cells are on same material type.

3. A node on the contact boundary - the adjacent cells contain particles of two and more material types.

4. Node on the free boundary - one from adjacent nodes is a "vacuum" node.

The dotted line on fig.3.3 designates the contact boundary between two substances their common free surface. Let's note that such classification of nodes resolves the boundary to within one cell size. 


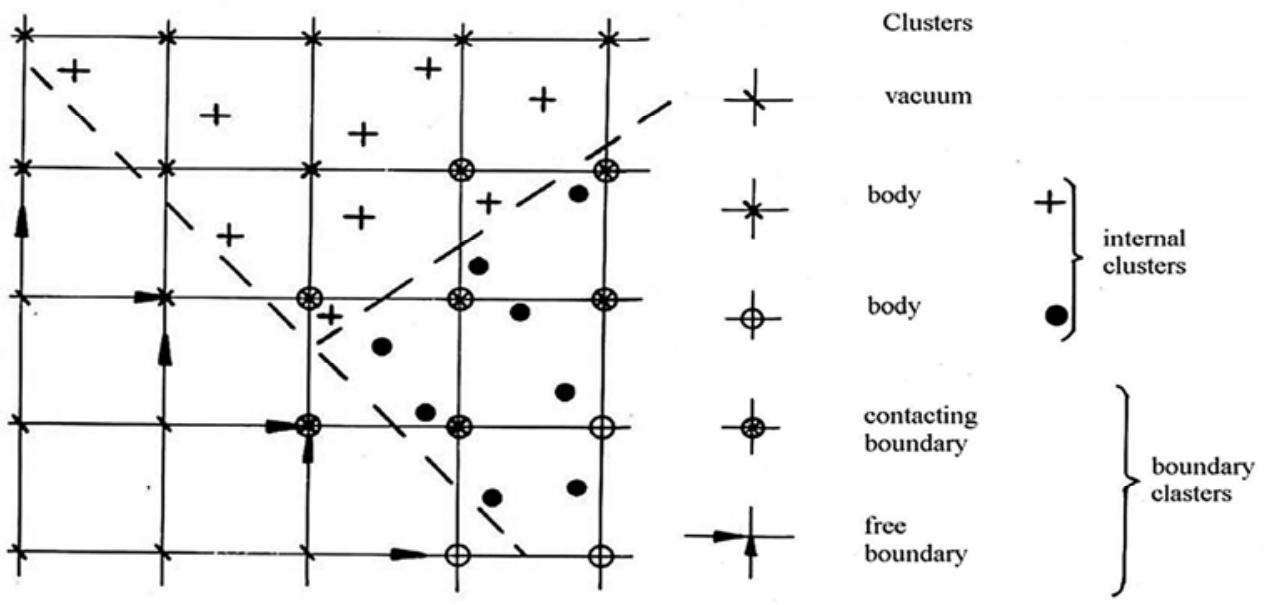

Fig. 3.3. Classification of nodes in Eulerian grid (the dotted line designates the boundaries of mixed fields)

Stage-II - statement of boundary conditions on the Eulerian grid (see below).

Stage-III - "grid to particle" interpolation and calculation of new values of particle parameters. The spatial derivatives are calculated at the nodes of the Eulerian grid (fig.2.4). In the interior nodes, all the derivatives are approximated by central differences, for example, in z-coordinate (two-sided derivative on fig.3.4):

$$
\left(\frac{\partial A}{\partial z}\right)_{i j}=\frac{A_{i+1, j}-A_{i-1, j}}{2 h} \text {. }
$$

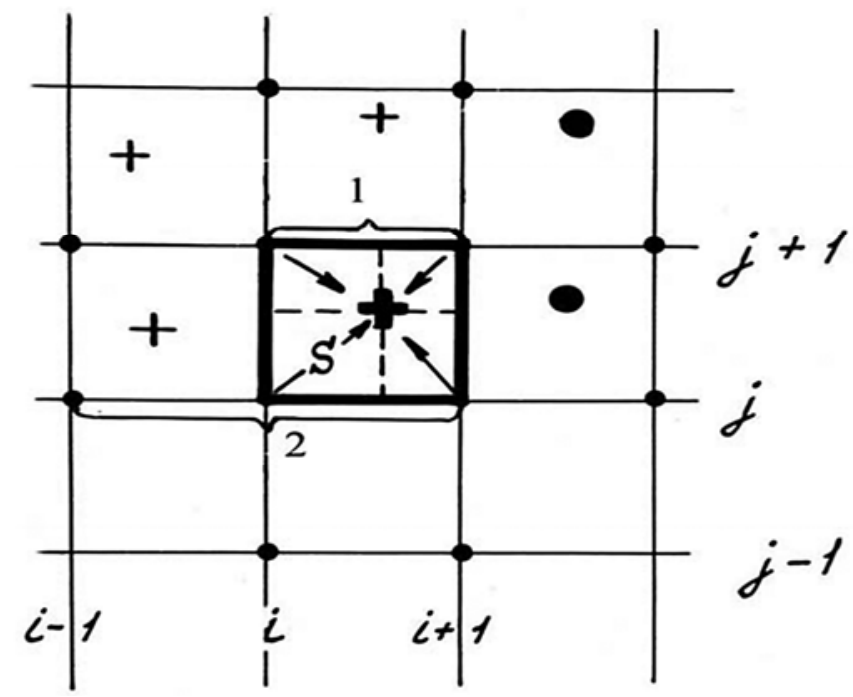

Fig. 3.4. 
In boundary nodes the calculation of derivatives of velocity components are carried out using one-sided difference formula, for example, the derivative of component Uin $\mathrm{z}$ coordinate is given by:

$$
\left(\frac{\partial U}{\partial z}\right)_{i, j+1}=\frac{U_{i+1, j+1}-U_{i, j+1}}{h} .
$$

Central difference scheme is used for the derivative of pressure. The values of derivatives at a particle are determined by linear interpolation of appropriate grid values as follows.

$$
\left(\frac{\partial A}{\partial z}\right)_{N}=\frac{\sum_{i j}\left(\frac{\partial A}{\partial z}\right)_{i j} S_{N}^{i j}}{\sum_{i j} S_{N}^{i j}}, \sum_{i j} S_{N}^{i j}=h^{2} .
$$

The summation is carried over the nodes. Here $\left(\frac{\partial A}{\partial z}\right)_{N}$ is the value of derivativesat particles, $\left(\frac{\partial \mathrm{A}}{\partial \mathrm{z}}\right)_{\mathrm{ij}}$ is the value of derivatives at nodes, $\mathrm{h}$ is the mesh size.

Thus obtained values of spatial derivatives at the particles are used for calculation at the next time step using the following difference scheme:

$$
\begin{gathered}
U_{N}^{k+1}=\widetilde{U}_{N}^{k}-\frac{\Delta t}{\rho_{N}^{k}}\left(\frac{\partial \tilde{p}}{\partial z}\right)_{N}^{k}, V_{N}^{k+1}=\widetilde{V}_{N}^{k}-\frac{\Delta t}{\rho_{N}^{k}}\left(\frac{\partial \tilde{p}}{\partial r}\right)_{N}^{k}, \\
z_{N}^{k+1}=z_{N}^{k}+U_{N}^{k+1} \Delta t, r_{N}^{k+1}=r_{N}^{k}+V_{N}^{k+1} \Delta t, \\
\rho_{N}^{k+1}=\rho_{N}^{k} /\left[1+(\operatorname{div} \vec{V})_{N}^{k} \Delta t\right], e_{N}^{k+1}=e_{N}^{k}-\frac{\widetilde{p}_{N}^{k}}{\rho_{N}^{k}}(\operatorname{div} \vec{V})_{N}^{k} \Delta t \\
q_{N}^{k+1}=\left\{\begin{array}{c}
-B c_{N}^{k} h(\operatorname{div} \vec{V})_{N}^{k}, i f \rho_{N}^{k+1} \geq \rho_{N}^{k} \\
0, \text { if } \rho_{N}^{k+1} \leq \rho_{N}^{k}
\end{array}\right.
\end{gathered}
$$

where divergence is defined as follows:

$$
(\operatorname{div} \overrightarrow{\mathrm{V}})_{\mathrm{N}}^{\mathrm{k}}=\left(\frac{\partial \mathrm{U}}{\partial \mathrm{z}}\right)_{\mathrm{N}}^{\mathrm{k}}+\left(\frac{\partial \mathrm{V}}{\partial \mathrm{r}}\right)_{\mathrm{N}}^{\mathrm{k}}+\left(\frac{\mathrm{V}}{\mathrm{r}}\right)_{\mathrm{N}^{\prime}}^{\mathrm{k}}
$$

The index $\mathrm{N}$ refers to a particle with number $\mathrm{N}$, $\mathrm{k}$ refers to the time step number, $\Delta \mathrm{t}$ is the time step size, $C$ is the velocity of a sound,Bis the constant for the given substance. The value of $B(\sim 1)$ used, depends on stability conditions requiring artificial viscosity. Let's note that in evaluation of velocity components $U_{\mathrm{N}}^{\mathrm{k}+1}, \mathrm{~V}_{\mathrm{N}}^{\mathrm{k}+1}$ at a particle, the right hand side of the appropriate formulas does not use the old values of velocity, rather the intermediate magnitudes $\widetilde{U}_{\mathrm{N}}^{\mathrm{k}}, \widetilde{\mathrm{V}}_{\mathrm{N}}^{\mathrm{k}}$, obtained by interpolating the nodal velocities. Fromconservation of total energy at a particle, the value of its specific internal energy in this case is modified by:

$$
\tilde{\mathrm{e}}_{\mathrm{N}}^{\mathrm{k}}=\mathrm{e}_{\mathrm{N}}^{\mathrm{k}}+\frac{\left(\mathrm{U}_{\mathrm{N}}^{\mathrm{k}}\right)^{2}+\left(\mathrm{V}_{\mathrm{N}}^{\mathrm{k}}\right)^{2}-\left(\widetilde{\mathrm{U}}_{\mathrm{N}}^{\mathrm{k}}\right)^{2}-\left(\widetilde{\mathrm{V}}_{\mathrm{N}}^{\mathrm{k}}\right)^{2}}{2}
$$

Thus the total energy and the momentum of the particle system do not vary. A "smoothing" procedure (by which, the average value of particles velocity is calculated) for particle velocity is required for every 5-10 time steps to ensure monotonicity of the solution. The smoothing at each time step reduces the spreading of shock wave to 2 to 3 cells. For the time 
step without smoothing the values $\mathrm{U}_{\mathrm{N}}^{\mathrm{k}+1}, \mathrm{~V}_{\mathrm{N}}^{\mathrm{k}+1}$ and $\mathrm{e}_{\mathrm{N}}^{\mathrm{k}}$ are used in (3.5) instead of the intermediate values of $\widetilde{U}_{N}^{k+1}, \widetilde{V}_{N}^{k+1}$ and $\tilde{e}_{N}^{k}$. The scheme (3.5) is conservative and is first order accurate in time and space.

In presence of elastic-plastic effects the system (2.1-2.17) is evaluated on some time step $\mathrm{k}$ as follows.

The values of momentum at particles are interpolated at nodes, and then subsequently nodal values of velocities are calculated using boundary conditions. Nodal values of derivatives of velocities are carried out. Then these derivatives are mapped onto the particles.

New density:

$$
\rho_{\mathrm{N}}^{\mathrm{k}+1}=\rho_{\mathrm{N}}^{\mathrm{k}} /\left[1+(\operatorname{div} \overrightarrow{\mathrm{V}})_{\mathrm{N}}^{\mathrm{k}} \Delta \mathrm{t}\right]
$$

a. New specific internal energy:

$$
\mathrm{e}_{\mathrm{N}}^{\mathrm{k}+1}=\mathrm{e}_{\mathrm{N}}^{\mathrm{k}}+\frac{\Delta \mathrm{t}}{\rho_{\mathrm{N}}^{\mathrm{k}}}\left\{\mathrm{P}_{\mathrm{N}}^{\mathrm{zz}}\left[\left(\frac{\partial \mathrm{U}}{\partial \mathrm{r}}\right)_{\mathrm{N}}+\left(\frac{\partial \mathrm{V}}{\partial \mathrm{z}}\right)_{\mathrm{N}}\right]+\mathrm{P}_{\mathrm{N}}^{\mathrm{rr}}\left(\frac{\partial \mathrm{V}}{\partial \mathrm{r}}\right)_{\mathrm{N}}+\mathrm{P}_{\mathrm{N}}^{\varphi \varphi}\left(\frac{\mathrm{V}}{\mathrm{r}}\right)_{\mathrm{N}}\right\}
$$

Here

$$
P_{N}^{i j}=-\left(p_{N}^{k}+q_{N}^{k}\right)+\left(S_{N}^{i j}\right)^{k}, \text { if } i=j, P_{N}^{z r}=\left(S_{N}^{i j}\right)^{k},
$$

b. Linear artificial viscosity:

$$
q_{N}^{k+1}=\left\{\begin{array}{c}
-B \rho_{N}^{k} c_{N}^{k} h(\operatorname{div} \vec{V})_{N}^{k}, i f \rho_{N}^{k+1}>\rho_{N}^{k} \\
0, \text { if } \rho_{N}^{k+1} \leq \rho_{N}^{k} .
\end{array}\right.
$$

c. New components of stress deviator:

$$
\begin{gathered}
\left(\mathrm{S}_{\mathrm{N}}^{\mathrm{zz}}\right)^{k+1}=\left(\mathrm{S}_{\mathrm{N}}^{\mathrm{zz}}\right)^{k}+\left\{2 \mu\left[\left(\frac{\partial \mathrm{U}}{\partial \mathrm{z}}\right)_{\mathrm{N}}-\frac{1}{3}(\operatorname{div} \overrightarrow{\mathrm{V}})_{N}\right]+\delta_{\mathrm{N}}^{\mathrm{zz}}\right\} \Delta t \\
\left(\mathrm{~S}_{\mathrm{N}}^{\mathrm{rr}}\right)^{k+1}=\left(\mathrm{S}_{\mathrm{N}}^{\mathrm{rr}}\right)^{k}+\left\{2 \mu\left[\left(\frac{\partial \mathrm{V}}{\partial \mathrm{r}}\right)_{\mathrm{N}}-\frac{1}{3}(\operatorname{div} \overrightarrow{\mathrm{V}})_{N}\right]+\delta_{\mathrm{N}}^{\mathrm{rr}}\right\} \Delta t \\
\left(\mathrm{~S}_{\mathrm{N}}^{\mathrm{zr}}\right)^{k+1}=\left(\mathrm{S}_{\mathrm{N}}^{\mathrm{zr}}\right)^{k}+\left\{2 \mu\left[\left(\frac{\partial \mathrm{U}}{\partial \mathrm{r}}\right)_{\mathrm{N}}-\left(\frac{\partial \mathrm{V}}{\partial \mathrm{z}}\right)_{\mathrm{N}}\right]+\delta_{\mathrm{N}}^{\mathrm{zr}}\right\} \Delta t
\end{gathered}
$$

Magnitudes of $\delta_{\mathrm{N}}^{\mathrm{ij}}$ - single-error correction due to rotation

$$
\delta_{\mathrm{N}}^{\mathrm{zZ}}=\left(\mathrm{S}_{\mathrm{N}}^{\mathrm{zr}}\right)^{k}\left[\left(\frac{\partial \mathrm{V}}{\partial \mathrm{z}}\right)_{\mathrm{N}}-\left(\frac{\partial \mathrm{U}}{\partial \mathrm{r}}\right)_{\mathrm{N}}\right], \delta_{\mathrm{N}}^{\mathrm{rr}}=-\delta_{\mathrm{N}}^{\mathrm{zz}}, \quad \delta_{\mathrm{N}}^{\mathrm{zz}}=\frac{\left(\mathrm{S}_{\mathrm{N}}^{\mathrm{rr}}\right)^{k}-\left(\mathrm{S}_{\mathrm{N}}^{\mathrm{zz}}\right)^{k}}{2}\left[\left(\frac{\partial \mathrm{V}}{\partial \mathrm{z}}\right)_{\mathrm{N}}-\left(\frac{\partial \mathrm{U}}{\partial \mathrm{r}}\right)_{\mathrm{N}}\right],
$$

d. The Von Mises yield criterion is satisfied as follows.

$$
J=\left[\left(S_{N}^{\mathrm{ZZ}}\right)^{\mathrm{k}+1}\right]^{2}+\left[\left(\mathrm{S}_{\mathrm{N}}^{\mathrm{rr}}\right)^{\mathrm{k}+1}\right]^{2}+\left[\left(\mathrm{S}_{\mathrm{N}}^{\mathrm{zr}}\right)^{\mathrm{k}+1}\right]^{2}+\left(\mathrm{S}_{\mathrm{N}}^{\mathrm{ZZ}}\right)^{\mathrm{k}+1}\left(\mathrm{~S}_{\mathrm{N}}^{\mathrm{rr}}\right)^{\mathrm{k}+1} .
$$

To satisfy the inequality $\mathrm{J} \leq \frac{1}{3} \sigma_{0}^{2}$ all terms of the stress deviator $\left(\mathrm{S}_{\mathrm{N}}^{\mathrm{ij}}\right)^{\mathrm{k}+1}$ are multiplied by on a factor $\sqrt{\frac{1}{3 \mathrm{~J}} \sigma_{0}}$. 
e. "Smoothening" of velocity and adjustment of specific internal energy is produced

$$
\begin{gathered}
\widetilde{\mathrm{U}}_{\mathrm{N}}^{\mathrm{k}}=\sum_{\mathrm{ij}} \mathrm{U}_{\mathrm{ij}} \Delta_{\mathrm{N}}^{\mathrm{ij}}, \widetilde{\mathrm{V}}_{\mathrm{N}}^{\mathrm{k}}=\sum_{\mathrm{ij}} \mathrm{V}_{\mathrm{ij}} \Delta_{\mathrm{N}}^{\mathrm{ij}}, \\
\tilde{\mathrm{e}}_{\mathrm{N}}^{\mathrm{k}+1}=\mathrm{e}_{\mathrm{N}}^{\mathrm{k}+1}+\frac{\left(\mathrm{U}_{\mathrm{N}}^{\mathrm{k}}\right)^{2}+\left(\mathrm{V}_{\mathrm{N}}^{\mathrm{k}}\right)^{2}-\left(\widetilde{\mathrm{U}}_{\mathrm{N}}^{\mathrm{k}}\right)^{2}-\left(\widetilde{\mathrm{V}}_{\mathrm{N}}^{\mathrm{k}}\right)^{2}}{2}, \text { where } \Delta_{N^{-}}^{\mathrm{ij}} \text { square of } S_{N}^{\mathrm{ij}} \text { (see Fig.2.2). }
\end{gathered}
$$

Here $\widetilde{U}_{N}^{k}, \widetilde{V}_{N}^{k}$ are the smoothed values of velocity components, $\tilde{e}_{N}^{k+1}$ is the corrected value of a specific internal energy. For each particles with known values of density and specific internal energy with a given equation of state the hydrostatic pressure $\mathrm{p}_{\mathrm{N}}^{\mathrm{k}+1}=\mathrm{p}\left(\mathrm{N}_{\mathrm{B}}, \rho_{\mathrm{N}}^{\mathrm{k}+1}, \widetilde{\mathrm{e}}_{\mathrm{N}}^{\mathrm{k}+1}\right)$ is calculated. Then the components of stress tensor $\mathrm{P}_{\mathrm{N}}^{\mathrm{ij}}=$ $-\left(\mathrm{p}_{\mathrm{N}}^{\mathrm{k}+1}+\mathrm{q}_{\mathrm{N}}^{\mathrm{k}+1}\right)+\left(\mathrm{S}_{\mathrm{N}}^{\mathrm{ij}}\right)^{\mathrm{k}+1}$ are calculated and are interpolated at the nodes. Then after taking into account of boundary conditions, the grid values of derivatives of stress tensor components are determined. For each particle, the new values of velocities and coordinates are calculated as follows.

$$
\begin{gathered}
\mathrm{U}_{\mathrm{N}}^{\mathrm{k}+1}=\widetilde{\mathrm{U}}_{\mathrm{N}}^{\mathrm{k}}+\frac{\Delta \mathrm{t}}{\rho_{\mathrm{N}}^{\mathrm{k}+1}}\left[\left(\frac{\partial \mathrm{P}^{\mathrm{zz}}}{\partial \mathrm{z}}\right)_{\mathrm{N}}+\left(\frac{\partial \mathrm{P}^{\mathrm{zr}}}{\partial \mathrm{r}}\right)_{\mathrm{N}}+\frac{\mathrm{P}_{\mathrm{N}}^{\mathrm{zr}}}{\mathrm{r}_{\mathrm{N}}}\right], \mathrm{V}_{\mathrm{N}}^{\mathrm{k}+1}=\widetilde{\mathrm{V}}_{\mathrm{N}}^{\mathrm{k}}+\frac{\Delta \mathrm{t}}{\rho_{\mathrm{N}}^{\mathrm{k}+1}}\left[\left(\frac{\partial \mathrm{P}^{\mathrm{zr}}}{\partial \mathrm{z}}\right)_{\mathrm{N}}+\left(\frac{\partial \mathrm{P}^{\mathrm{rr}}}{\partial \mathrm{r}}\right)_{\mathrm{N}}+\frac{\mathrm{P}_{\mathrm{N}}^{r r}-\mathrm{P}_{\mathrm{N}}^{\varphi \varphi}}{\mathrm{r}_{\mathrm{N}}}\right] \\
\mathrm{z}_{\mathrm{N}}^{\mathrm{k}+1}=\mathrm{z}_{\mathrm{N}}^{\mathrm{k}}+\mathrm{U}_{\mathrm{N}}^{\mathrm{k}+1} \Delta \mathrm{t}, \mathrm{r}_{\mathrm{N}}^{\mathrm{k}+1}=\mathrm{r}_{\mathrm{N}}^{\mathrm{k}}+\mathrm{V}_{\mathrm{N}}^{\mathrm{k}+1} \Delta \mathrm{t} .
\end{gathered}
$$

With this the calculation for the next time step is completed. After the first stage of calculation in the IP method the values of pressure and velocity components are known at the nodes. The type of each node - "vacuum", boundary or interior is also known. It is possible to divide boundary conditions on the Eulerian grid into two groups (implementation of which is carried out at the second stage of calculation): 1) Boundary conditions of a designated field (e.g. a rigid inclusion) and 2) conditions on free and contact surfaces.

Let's consider boundary conditions on a designated boundary. In that case, when the boundary is a rigid wall or line of symmetry, the boundary conditions for a continuous medium require that the flow should be parallel to the boundary, that is, the normal component of velocity should be equal to zero. We use a method of reflection for such cases. Exterior to the boundary additional sets of fictitious nodes are introduced, which contain anti-symmetric reflection of normal velocity components, and symmetric reflection of remaining variables. So the following values of parameters are set in fictitious meshes for non-reflecting rigid boundary or for the lines of symmetry.

$$
V_{j-1}=-V_{j+1}, U_{j-1}=U_{j+1}, \tilde{P}_{j-1}=\tilde{P}_{j+1} .
$$

Condition $V_{j}=0$, here index $j$ refers to the set of nodes on the boundary, $j-1$ refers to fictitious nodes, $j+1$ refers to nodes adjacent to the boundary in the interior. The linear extrapolation of parameters in the fictitious nodes is carried out using the condition of outflow of substance from the interior area as follows.

$$
A_{j-1}=2 A_{j}-A_{j+1}, A=(\tilde{p}, U, V) .
$$

In both cases (rigid wall and axis of symmetry), the type of node (interior, contact etc.) is also transmitted to the fictitious nodes. 


\section{Special algorithms to treat boundary nodes and particle shapes}

Before implementing boundary conditions along contact and free surfaces, we must know the spatial orientation of boundary surfaces. To achieve this, we use a single normal vector $\bar{N}$ that assigns orientation of the boundary surface at each node on the (pre-marked) free surfaces and contact boundaries. For contact boundaries, the normal vector is a normalized sum of vectors directed from the node under consideration to eight nearest nodes after taking into account their material contents. For contact surfaces, the normal vector is the normalized sum of vectors directed from the node under consideration to the nearest vaccuum nodes. For example, in figure 4.1 the vector normal to free boundary at node $(i, j)$ is the average of vectors from $\mathrm{N}_{\mathrm{ij}}$ to $\mathrm{N}_{\mathrm{i}-1, \mathrm{j}+1}, \mathrm{~N}_{\mathrm{i}-1, \mathrm{j}, \mathrm{j}} \mathrm{N}_{\mathrm{i}-1, \mathrm{j}, \mathrm{-}-1}, \mathrm{~N}_{\mathrm{i}, \mathrm{j}-\mathrm{1}-1}$.

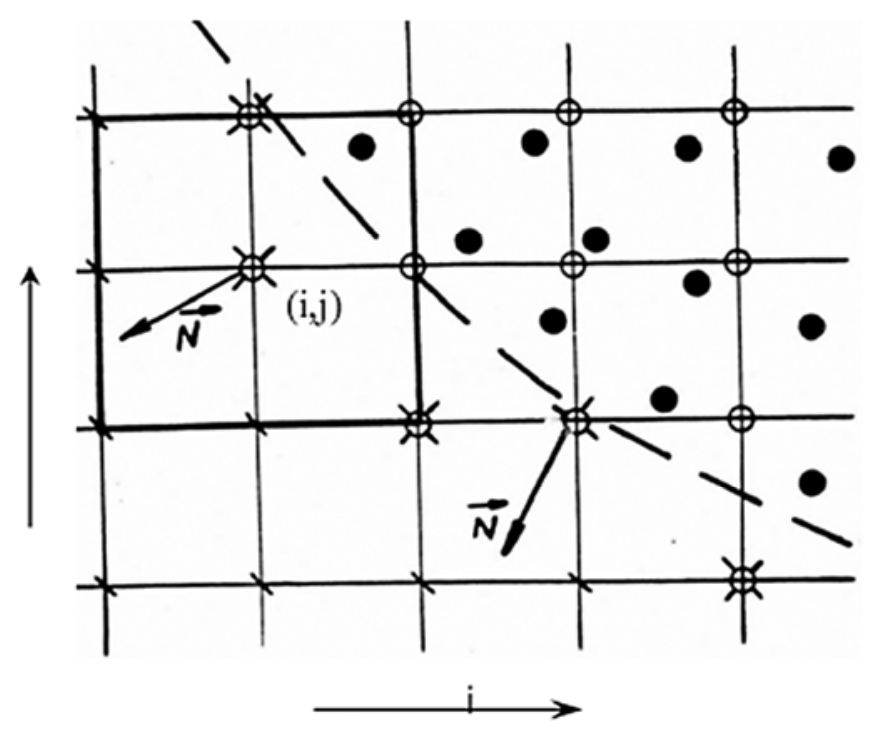

Fig. 4.1. Orientation of a normal vector $\overline{\boldsymbol{N}}$ on free surface (dotted line)

This procedure permits the determination of normal direction at boundaries within an accuracy of $\Pi / 8$. In most cases, the accuracy can be enhanced by using normal directions at adjacent boundary nodes. In fig.4.2 an example of the application of this algorithm is shown for the particle configuration discussed in figure 3.1. At each particle on the boundary, a line is drawn through the particle center and normal to the normal vector. The thicker lines pertain to the contact boundary.

On the free surfaces, the normal component of stress vector is set equal to zero. For gasdynamics calculations, the pressure is set equal to zero at the nodes on free surfaces. The sliding contact condition is enforced by recalculating the velocity components at particles such that the normal velocity component remains unchanged.

$$
\begin{aligned}
& \bar{U}=U_{i j} N_{z}^{2}+U_{N} N_{r}^{2}+\left(V_{i j}-V_{N}\right) N_{z} N_{r}, \\
& \bar{V}=V_{i j} N_{z}^{2}+V_{N} N_{r}^{2}+\left(U_{i j}-U_{N}\right) N_{z} N_{r}
\end{aligned}
$$


Where $U_{\mathrm{ij}}, \mathrm{V}_{\mathrm{ij}}$ - velocity components on contact boundary, $\overline{\mathrm{U}}_{\mathrm{ij}}, \overline{\mathrm{V}}_{\mathrm{ij}}$ - appropriate recalculating value, $N_{z}, N_{r}$ - component of normal vector at ij th node, $U_{N}, V_{N}$ - velocity components at $N^{\text {th }}$ particle.

When the tensile stress at a node on contact boundary exceeds some critical value $\mathrm{p}^{*}$, a free boundary is introduced through the node, and the particle's normal stress component is set equal to zero. Thus a possible separation of materials along contact boundary is facilitated. The choice $\mathrm{p}^{*}$ is arbitrary. Good results are obtained by using a value that is small (by two orders of magnitude) compared to the characteristic stress in the flow field.

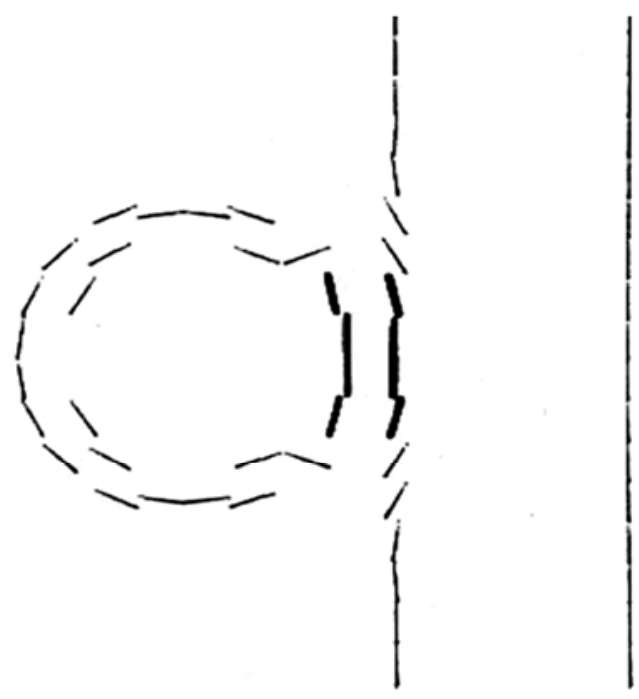

Fig. 4.2. An example of application of algorithm to determine boundaries for the configuration of particles represented on fig.3.1. (Thick lines show contact boundary)

During the calculation, some particles may cross over rigid boundaries. For such cases, a distance $l_{\min }$ is calculated based on the mass and density of the particles. When a particle reaches within this distance to the rigid boundary, its normal velocity component $V_{\overrightarrow{\mathrm{n}}}$ is set equal to zero, and the internal energy is raised by $\Delta e=V_{\vec{n}}^{2} / 2$ which equals the loss of kinetic energy due to velocity adjustment. The velocity component along the rigid boundary does not change. In case of outflow of substance through the open boundary (prescribed as calculation limit) the particle does not participate in further calculations. As the calculation proceeds in the IP method, there may arise a situation where empty (Eulerian) cells occupy the space between cells containingparticles. (i.e. some cells do not contain any particles). Such cases arise in zones of large expansion and in zones experiencing large strains. In such cases, some particles cease "to feel" each other as the area of influence of each particle in limited to the cell where it resides. This is a typical characteristic of particle in cell methods. One elementary way to eliminate this problem is to use large number of particles initially in the areas where such problem may potentially occur. But this is a cumbersome and inefficient way as it is not always possible to foresee where such problems may occur. A more rational means to eliminate such problems arising out of discontinuity is presented here. 
For monitoring local violations of continuous presence of particles in each cell, new parameters are included to describe a particle in vector form (fig. 4.3). In planar symmetry case, the particle represents a rectangular parallelepiped of unit length, while for axisymmetric case it is a torus. The projection of a parallelepiped or a torus on $(\mathrm{z}, \mathrm{r})$ plane is a rectangle. Stretch and rotation of the rectangle are defined by components of a vector, which determines the orientation of the particle. The two sides of the rectangle, $a$ and $b$ are related to the vector components $\mathrm{L}_{\mathrm{z}}$ and $\mathrm{L}_{\mathrm{r}}$ as follows.

$$
\mathrm{a}=\sqrt{\mathrm{L}_{\mathrm{z}}+\mathrm{L}_{\mathrm{r}}}, \mathrm{b}=\frac{\mathrm{M}}{\mathrm{da} \rho},
$$

Where the parameter $\mathrm{d}$, having dimension of length, is equal to 1 for planar symmetry, and is equal to $2 \pi r_{N}$ for rotational symmetry $\left(r_{N}-r\right.$ coordinate of particle center). The orientation of the triangle with respect to the $\mathrm{z}$-axis is given by,

$$
\varphi=\operatorname{arctg}\left(\frac{L_{z}}{L_{r}}\right) .
$$

The vector components $L_{r}$ and $L_{z}$ evolve with time as follows:

$$
\frac{\mathrm{d} L_{z}}{\mathrm{dt}}=\frac{\partial \mathrm{U}}{\partial \mathrm{r}} L_{r}+\frac{\partial \mathrm{U}}{\partial \mathrm{z}} L_{z}, \frac{\mathrm{d} L_{r}}{\mathrm{dt}}=\frac{\partial \mathrm{V}}{\partial \mathrm{r}} L_{r}+\frac{\partial \mathrm{V}}{\partial \mathrm{z}} L_{z} .
$$

The interpolation of velocity and pressure to Eulerian grid is carried out from the center of individual particles, irrespective of their form or orientation.

During transition of a particle in a flow field it can (not changing the form of a rectangle):

1. Stretch and rotate,

2. Increase in size without changing shape, for example, during dilatational wave.

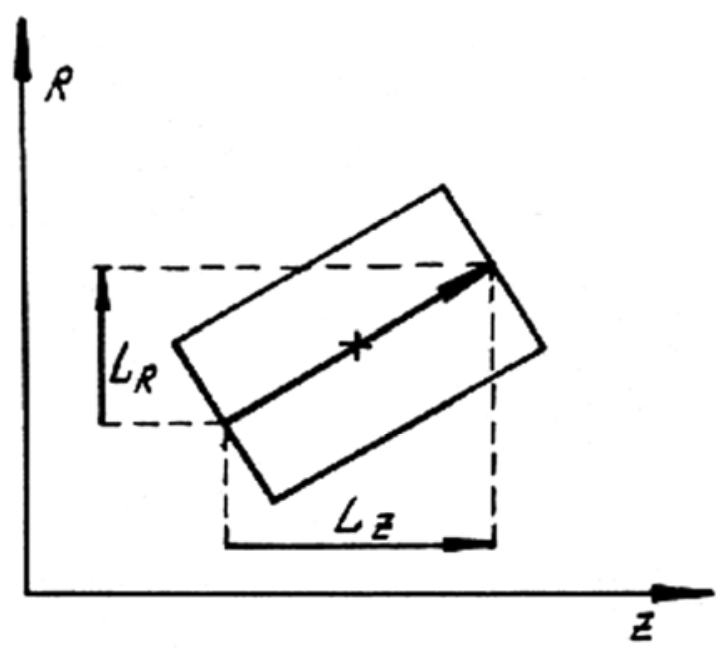

Fig. 4.3. The vector-form of a particle

In both cases continuous distribution of particles (at least one particle per cell) may be disrupted. Empty cells may arise in the direction of stretching for the first case above, or in the orientation directions of its sides as in the second case. It is natural to entertain virtual 
particles ("virtual" - because the parameters of these particles are not stored in the computer memory), i.e., to assume that the base particle consists of two (for case-1) or four (for case-2) particles. The virtual particles have equal mass whose sum is equal to the mass of the base particle. The remaining parameters, other than the coordinates, at the virtual particles are identical. Thus, the interpolation at the nodes is carried out from one base particle, two (for case-1) virtual particles or four (for case-2) virtual particles. The virtual particles are introduced based on the value of a dimensionless number $\eta$. The base particle is substituted by two virtual particles when $a / b>\eta$, and by four particle when $a / h>\eta$, where $a$ and $b$ are the larger and smaller sides of the rectangle respectively, and $h$ is the node spacing. The location of virtual particles inside a base particle is determined from their rectangular distribution in the domain. For most practical calculations the value of $\eta$ starts at 1.2. For larger values the quality of observance of continuous distribution of particles in cells worsens, while for smaller values the computational time increases due to emergence of large number of virtual particles.

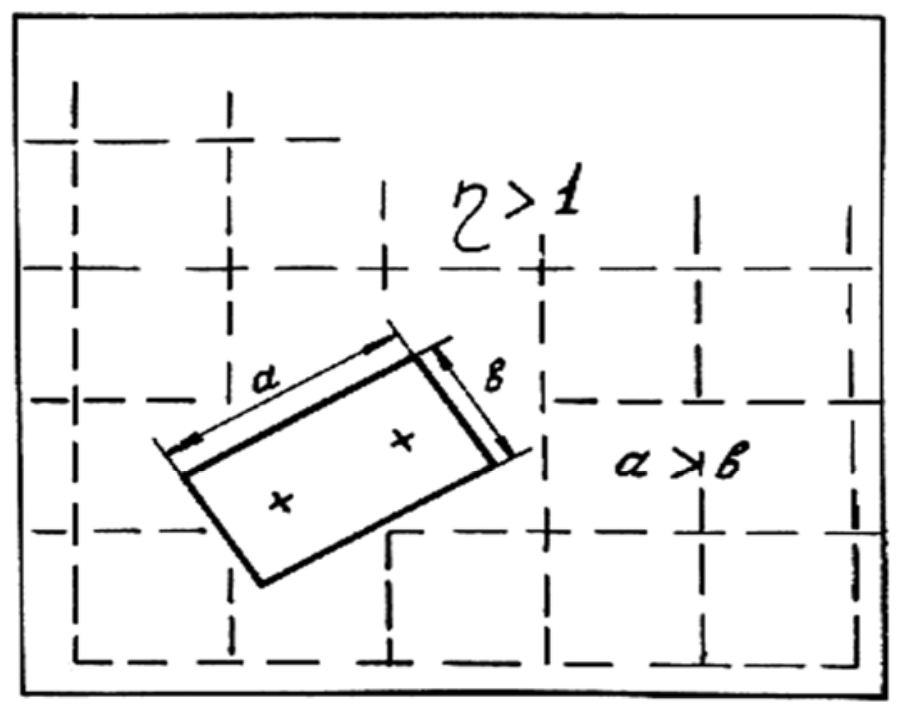

Fig. 4.4. Formation of virtual particles

In the event that the particle is stretched to twice its original size (or the size of virtual particle equals the original size of the base particle), it is better to divide the base particle into two particles. As a result, the virtual particles become real particles, and the computer memory is accordingly adjusted.

An example illustrating how virtual particles form is showed in fig.4.5. A bar of aluminum impacts a rigid wall at $1 \mathrm{~mm} / \mu$ s. Planar symmetry is used. Cells of $1 \mathrm{~mm} X 1 \mathrm{~mm}$ size are used in the Eulerian grid. The bar is $5 \mathrm{~mm}$ along z-axis and $10 \mathrm{~mm}$ along r-axis. The left boundary is the axis of symmetry. Distribution of horizontal component of velocity is shown at $10 \mathrm{~ms}$. The dotted lines show discontinuity in solution when no account is taken of the form of the particles. The solution in such case does not describe actual physical situation. On the other hand, when the form of the particles is taken into account and the particles are allowed to subdivide, the discontinuity in the solution disappears. In the figure $4.5 \mathrm{~b}$, zones 
of various levels of strain are depicted by degree of stretch, rotation, and subdivision of particles. This provides additional information about singularities in solution. Near free surface, where strain is insignificant, particle subdivision does not take place. Thus by introducing virtual particles algorithm in the IP method, automatic adaptivity is achieved that enables capture of singularity in the solution.
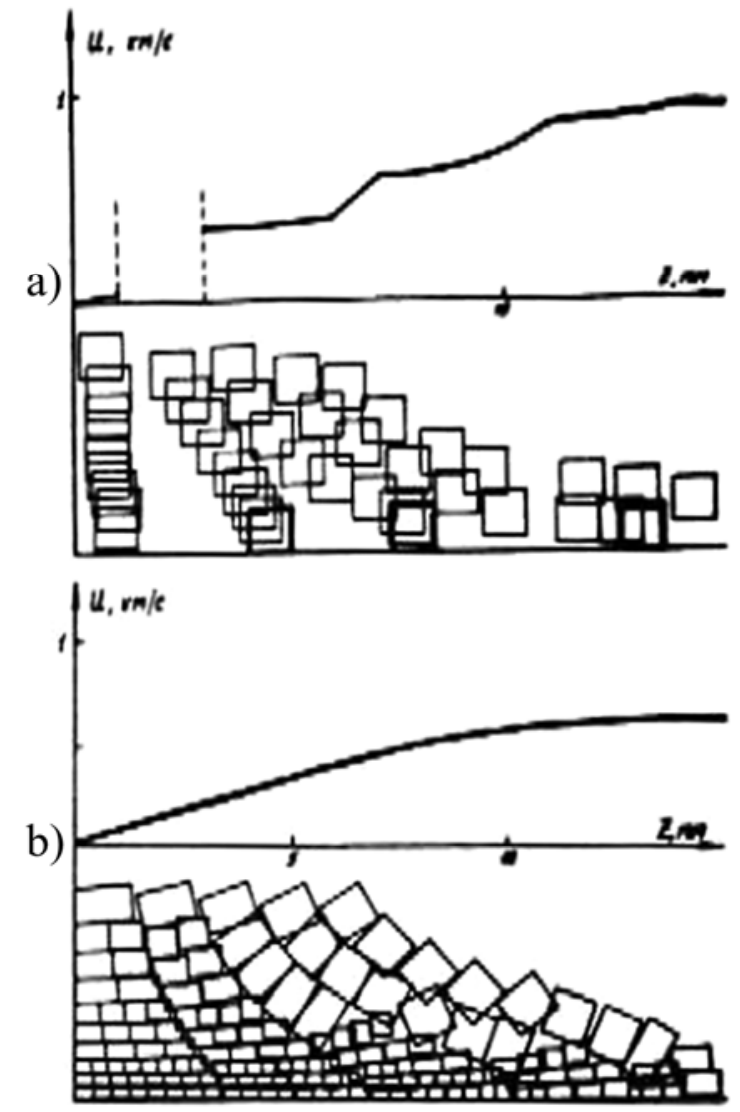

Fig. 4.5. An aluminum bar impacting a rigid wall. a) Without accounting for the form of particles, b) Accounting for the form of particles and their subdivision. The dotted line indicates disruption of continuity of the velocity profile along the wall

\section{Examples}

The basic aspects of the technology of the computational experiment (CE), principles of program-technical systems (PTS) construction, developed under the scientific leadership of Professor Vladilen F. Minin (Novosibirsk, USSR) on the basic of experience of development and usage of similar systems for 35 years and designed for realization of mass computational experiment are formulated in the given work. The main parameters of PTS 
are yielded, some most interesting trends of CE conduction are shown, each of them is illustrated with the relevant solved problems.

The development of the mathematical physics, computational mathematics and means of computer technology gives the possibility to analyze the processes of pulse action upon a substance by conducting the computational experiment. It should be noted that in a general case the notion of $\mathrm{CE}$ is wider than the notion of simple modulation. The main difference is in the fact that CE makes possible to answer the question: "What will be if ...?"

On the basis of experience of development and usage of PTS the following main principles of PTS construction were formulated [4-5]:

- the problem solution can be carried out by a researcher who is not a specialist in the region of numerical methods and programming;

- $\quad$ PTS operate in a dialogue regime in terms of a researcher;

- all the initial and resulting information is represented in a convenient from analysis and displayed, that is the principle of "no dumps" is realized;

The PTS satisfying the requirements given above was realized using two 64-processor computers on the basic of the multiprocessor PS-2000 and a serial graphic display station. For complete realization of multiprocessor possibilities a special code allowing to carry out its paralleling was developed to solve the equation of continuum mechanics. For the solution of a wide class of problems besides generally used mathematical models of description of material and substance properties the wide-range equations of state of metals and description models of nonstationary detonation of heterogeneous explosives taking into account the kinetics of their decomposition in shock waves were included in PTS.

The main parameters of PTS are as follows: the RAM capacity is 2000000 24-digit words, the calculation field size is to 200000 units per one step in time, the calculation time of one step is 6-8 s, the average calculation time of one variant of the problem using the method of individual particles [6] was 2-3 hours.

A computation experiment on the computer complex was conducted in the following sequence [6]:

- the required and achievable space resolution, the time step, the regime of the algorithm operation, the boundaries of the calculation configuration variation and so on were defined in the first phase;

- the calculation of the subsequent variants was carried out in the regime of recording of a display-film, that is, the information about the process run was recorded on the magnetic disks with a sufficiently short time step which practically excluded the possibility of the data loss about the details of the process. But at the same time numeric data were not stored only the packed graphic information about the dynamics of the process occupying not much disk space was recorded. The character of the flow configuration change and the parameter change was shown by means of the colour palette and different grades of brightness for each colour. The velocity of look of the display-film with the frame size about 50000 pixels was about one frame per second.

- for obtaining the total numeric information about the run of the process the most typical and important (from view point of the investigator) variants are rerun once more in the regime of the full recording. The processing of the obtained frames in this case is carried out with displaying of the graphic information about the behavior of an arbitrary parameter $(\mathrm{P}, \rho, \mathrm{e}, \mathrm{U}$ or $\mathrm{V})$ in an arbitrary point of the calculation field. 
The wave configurations of the SW were discriminated and analyzed in the frames of the our computer complex by three techniques [7]: using a differential analyzer, by the maximum of the artificial viscosity - the values proportional to the pressure gradient and by pressure maximums in this cross-section of the calculation region. The first two techniques are essentially equivalent.

We should note that the technique of the differential analyzer allows to discriminate zone of fast change of calculated values which are associated with the SW fronts. The width of such zones in calculations is not more than 3-5 cells. Localization of the SW front (about 1 cell) is made by the line selection on which the velocity of change of the relevant calculated parameter is maximum.

Maximum pressure discrimination of wave fronts is made on the basis of the assumption that the transition from the incident wave to the reflected wave is accompanied by the normal deflection of the maximum locations to the axis $\mathrm{Z}$ to the side inverse to the front of the incident SW. Such an analysis allows to discriminate the location of contact points of two SW with the precision to one cell. The calculated value of the reflection (incidence) angle of the SW is defined by the average slope of the front of the reflected (incident) wave almost over 5 cell.

The development of the basis of the technology of CE conduction and long-term usage of the created PTS allowed carrying out a series of CE and obtaining new knowledge in the following main directions [8]:

- Simulation of cosmogeneous bombardment of the Earth and the project of the "space protection" of the planet, problems of the shock-explosive tectonics [9]. The system of equations describing the dynamics of the volume increase of microcracks (microvoids) was used in simulation of impact. It could be noted that the problems connected with formation of a crater and with movement of the ground in the explosion of a meteorite near the surface or upon collision with the ground are of constant interest. The basic mechanical properties characterizing this process are the parameters of the shock wave formed in the ground and the pattern of permanent displacements produced (in particular, the dimensions of the crater formed). The possibility of estimating shock-wave parameters for an explosion near the surface using known relationships for underground explosions has been discussed several times. The question of defining parameters for pulse application to some point of the surface of a semispace remains quite complex. As has been noted previously [10], cavity growth in electrical explosion on the surface of water proceeds according to a law close to the law of self similar motion at constant energy. It seems it was pioneer work because this fact allow to introduce the energy transferred to the ground and estimate its value (perhaps somewhat arbitrarily) by comparing the mechanical action parameters of contact and slightly submerged charges.

- dynamics simulation of high-speed collapses of dust particles of the Galley comet with protective screens of space vehicles in the project "Vega" [11-12], Fig.5.1; The widediapason equation of state was used. Also the computer simulation of the cumulative effect due to high - velocity $(1-20 \mathrm{~km} / \mathrm{s})$ impact interaction of projectiles with different thin screens and massive targets, arranged behind the screens, as well as of the destruction processes in these systems taking into account thermo-physical and reological properties of real constructive materials were investigated. The results of the computer simulation were in a good agreement with both the available experimental 
data and the analytical estimations in terms of a hydrodynamical theory of cumulation.
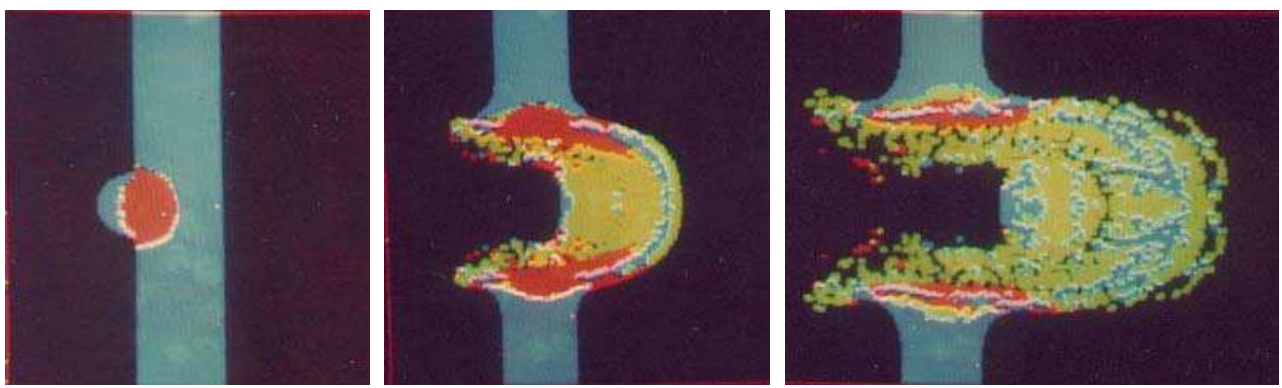

Fig. 5.1. Simulation of the macroparticle impact (V $30 \mathrm{~km} / \mathrm{s})$ with aluminum screen: blue solid, red - liquid, green - plasma states of matter

- work optimization of conical thermonuclear targets in the section of simulation of interaction of flat plates with a conical cavity [13];

- investigation of functioning of different constructions of generators of powerful Mach shock waves $[14,15]$ - Fig. 5.2.; These devices utilize the effect of geometric cumulation under conditions of irregular (Mach) reflection of conically convergent shock waves and are characterized by an additional concentration of energy during the shock wave convergence to the axis of symmetry and, at the same time, by an increased stability of flow as compared to the spherical convergence of shock waves.

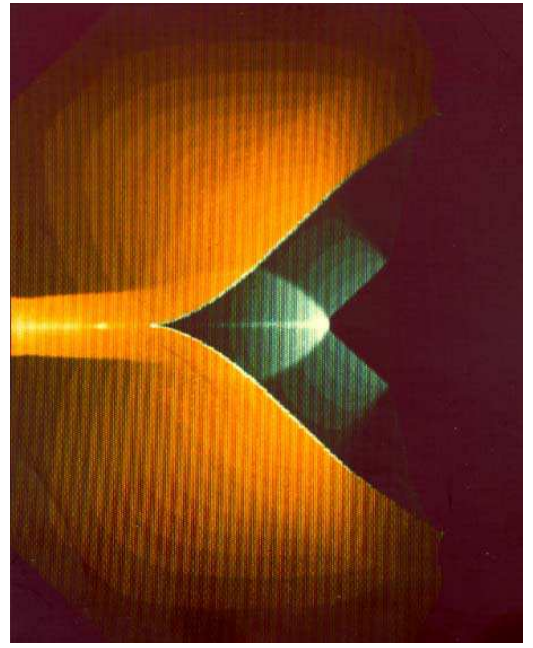

Fig. 5.2. Shock wave configuration on loaded conical target

- The peculiarities of the processes occurring on the shock-wave front and its stability were studied, and a new way to synthesize diamond in a shock- and detonation wave was developed as well as the way to synthesize wurtzite-like boron nitride widely used in industry (Fig.5.3). 

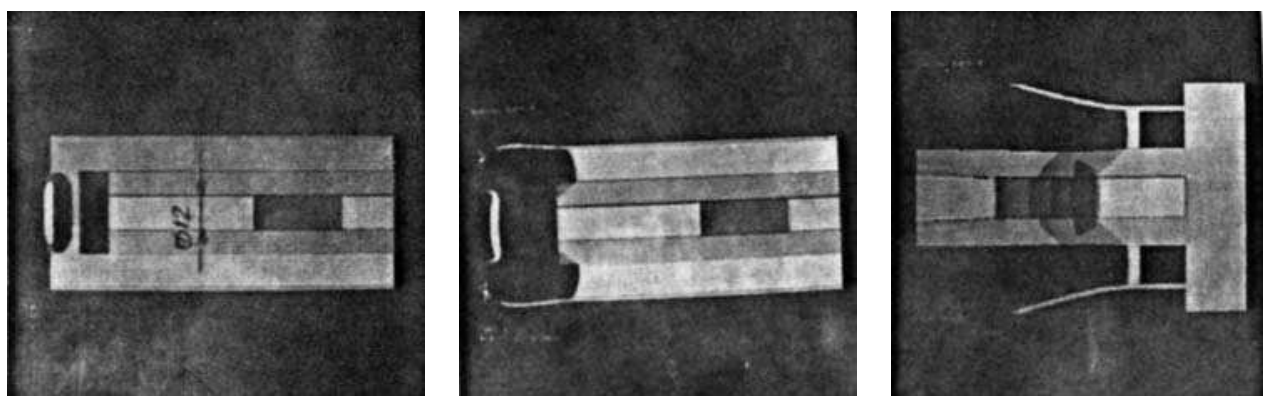

Fig. 5.3. Dynamic loading and Mach shock wave formation to synthesize diamond and wurtzite-like boron nitride

- development and simulation of the generation principle of powerful shock in a target while exposing it to space-modulated high-energy radiation [16];

- development and simulation of a new regime of a forced jet formation of high-speed plasma cumulative plasma jets (PJ) for thermonuclear projects while exposing profiled targets to radiation [17], fig. 5.4.;

It can be shown [17] that for the suggested regime of Jet formation in the first approximation

$$
\begin{aligned}
& V_{\max } \sim \operatorname{ctg}(\alpha) \sqrt{1+2 C_{f} \frac{x}{r_{0}},} \\
& x=(1-\cos (\alpha+\beta)) /((2+\cos (\alpha)-\cos (\alpha+\beta)) \sin (\alpha),
\end{aligned}
$$

where $\alpha$ - is a semiangle of the cone, $\beta$ - is an angle between the plate and the symmetry axis, $\mathrm{C}_{\mathrm{f}}$ - a coefficient of friction at the PJ propagation in a dense medium.

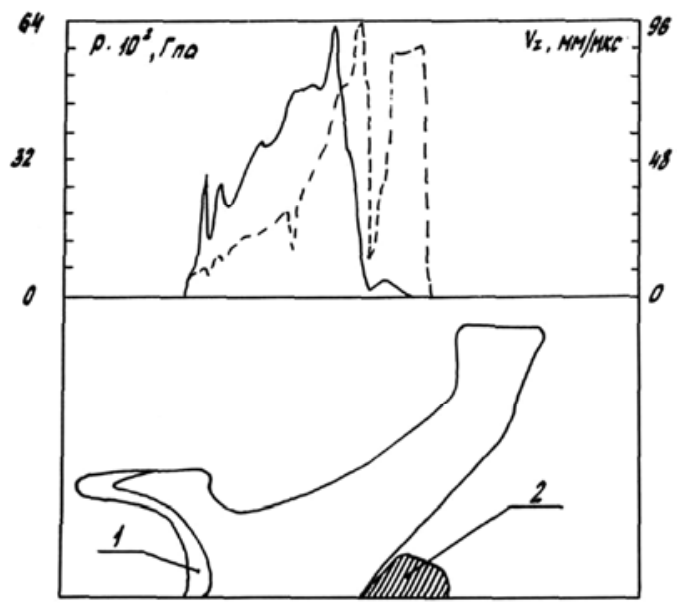

Fig. 5.4. Plasma forced jet formation in a conical target: 1 - pushed plate, 2 - plasma jet

It has been shown the realization of the forced Jet formation principle allows to increase both the speed and the pulse of the formed plasma jet essentially. More detailed analysis of the analytical model of non-stationary formation of the PJ shows that at the propagation of 
PJ not in vacuum but in the mediun with a low coefficient of resistance, as mentioned above, there is the effect of some increase of the PJ speed. And this medium as preliminary numerical experiments show makes a stabilizing action on the radial expansion of the PJ. It could be noted that as it is well known that the necessary requirements for the lack of a cumulative jet are established via the non-stationary collapsing of a liner of a cumulative hole. It has been also shown [18], that in a rotationally symmetric case the criterion by Wolsh is inapplicable. It was demonstrated that a rotationally symmetrical case that is produced by a current associated with a symmetrical axis produced by a shock wave can never be realized.

- a series of CE on studying of shock wave focusing possibilities into the space area of an arbitrary form in nonlinear regime by means of diffraction optics "taken" from the optical region into the continuum mechanics [19-20], fig.5.5.
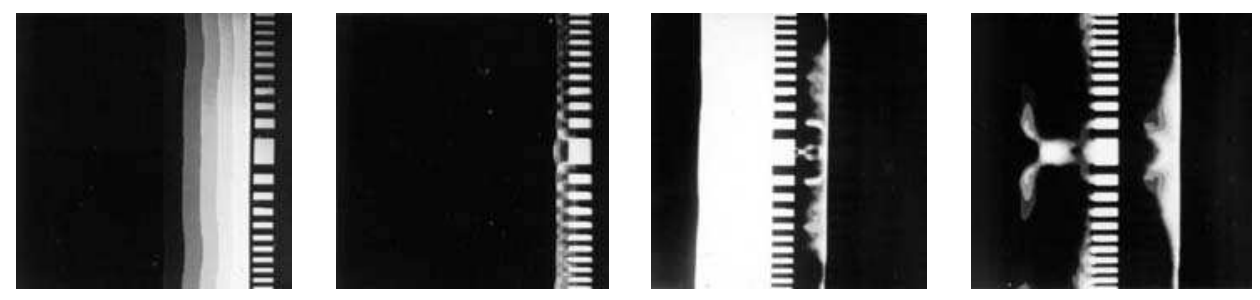

Fig. 5.5. The dynamics of shock wave focusing into the ring by means of diffractive element

- Numerical simulation of explosive plasma antenna [21]. For example, pulsed power generators as a sources are made on the magnetic cumulative generator or flux compression generator (FCG) bases the task of decreasing of dimensions and weight of them keeps being relevant. FCGs use the chemical energy from high explosives to accelerate a metallic conductor that traps and compresses a magnetic field initially created by a seed energy source such as a capacitor bank, battery, or another pulsed generator (figure 5.6).

The numerical experiments were also based on the following program complex, developments under the scientific leadership of Prof. Vladilen Minin:

- 3D supersonic aerodynamics [22],

- new methods of a modified large particles [23] allows to calculate explosive physics problems,

- The mathematical modeling of joint and magnetic-pulse welding processes [24],

- The mathematical modeling of castle. For example, in [25] the process of solidification and melting of the rod are described in the approximation of quasi-phase zone. Thermophysical parameters of the rod, casting and forms are different and equal to their average values in the range of temperatures.
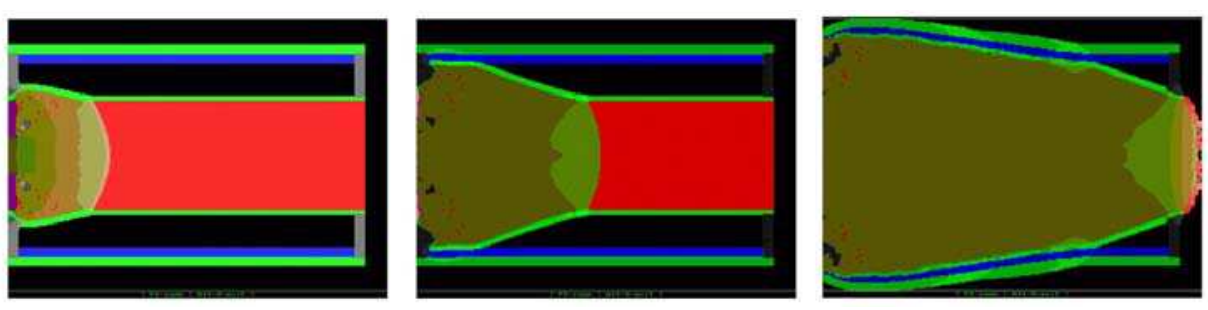

Fig. 5.6. Simulation of the FCG operation in dynamic [21] 
A direct experimental analysis of the dynamics of the form of pulsating gaseous cavities prof.V.F.Minin [26] discovered that under the action of shock wave a bubble collapses asymmetrically with the formation of a cumulative jet, which forms in the process of collapse and causes fragmentation of the bubble. It could be noted that in the paper [27] the cumulative jet or jet at all was not mentioned. There is no jet and on the experimental photographs presented in the paper. Moreover, when the dimension of gas bubble diameter of $0.7 \ldots 2 \mathrm{~cm}$, it was placed in a thin rubber membrane. Therefore, direct observation of the jet formation during compression of a gas bubble under the shock wave in the said article was not fulfilled. Experiments on the analysis of the attenuation of waves in bubble screens with different acoustic properties also were begun by V.F.Minin (1958), who examined also the first convenient model of a screen - a sequence of alternating flat one-dimensional liquid and gas layers.

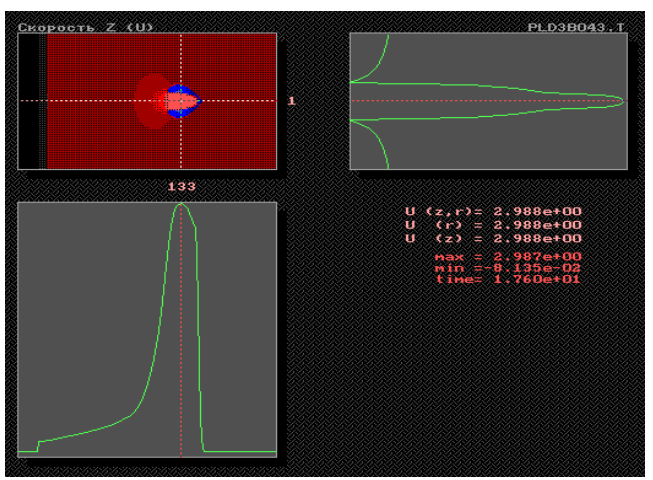

Fig. 5.7. Cumulative jet formations on air bobble collapsing under the shock wave: upper screenshot of jet formation (the axial velocity is shown), right - distribution of axial velocity along horizontal axis, bottom - distribution of axial velocity along vertical axis

The accumulated potential energy and energy of external field is converted as a result into the energy of cumulative jet. A jet pierces the cavity and this leads to the destruction of the cavity.

The maximum speed of cumulative jet exceeds the maximum speed of compression and it is more than the maximum speed of forward motion of cavity without taking into account deformation. The conversion of kinetic energy of compression into the kinetic translational energy does occur and later on, after the deformation, it converts to the kinetic energy of stream.
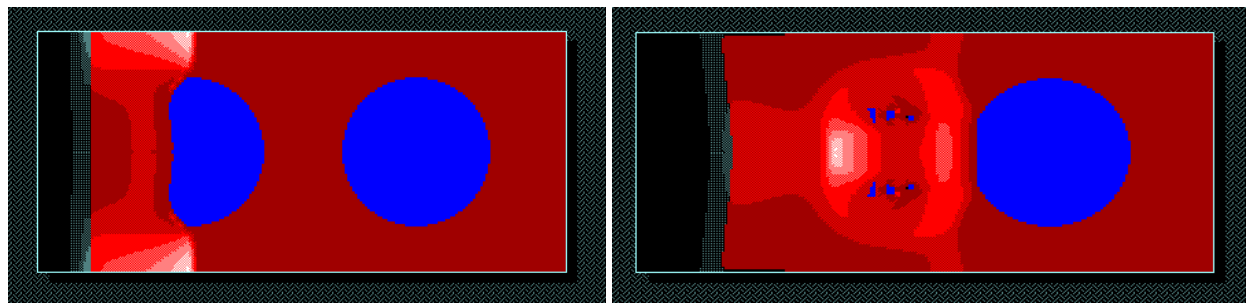

Fig. 5.8. Bubble collapsing and pulsation 
The process to form a jet for a bubble in cavitation field was also investigated. The sound field radiated by a vibrating bubble near a rigid (or a soft) boundary can be regarded as a superposition of the field of two bubbles which carried out in- (or out-) phase vibration when the boundary disappears. A numerical computation shows that in the neighborhood of its compressed phase, a compressed quasi-shock wave forms in it, which will be reflected at its center and becomes an expanding quasi-shock wave, then a large negative curvature or a very strong negative surface tensile will be produced suddenly in the part of the deformed bubble's surface where the reflected shock arrives there first, so that a jet will be formed, the top of which will point the rigid boundary (or back from the soft boundary). A calculation shows also the jet could generate new shock and may cause bubbles nearby to form new jets or to produce a chain-like reaction. The forces of surface tension are not considered in simulation.

\subsection{Hypercumulation - cumulative charges for efficient perforation of boreholes [28]}

The effect of cumulation at collision of liner with angles larger than 90 degrees from the axis of symmetry and corresponding compressing jets we called 'Hypercumulation' due to the fact that this process significantly increases energy content of a 'cumulative jet' and practically all of the liner material turns into high speed body - a 'jet' which can perform the required work. In experiments with liners at larger opening angles first occurs the process of classic cumulation with the production of a slug and a jet complying with normal cumulative flow and only after the liner passes the angle of 90 degrees a hypercumulation flow starts and the flow of cover metal is mostly transported into a cumulative jet, whose mass starts growing. This effect increases as increases collision angle and by a certain moment metal flow into the slug practically stops. Thus in this case we have two processes classic at the beginning and hypercumulative - some time later. It is necessary to note that both these processes are well detectable in X-rays pictures by many authors.

We managed to realise a hypercumulative process both in its pure form (Fig. 5.9) and in combination with a classic process and, what is the most important, we managed to regulate the maximal speed of the forefront part of the jet voluntarily.

This allows creating necessary speed gradients in order to stretch the jet and thus increase its efficiency, as well as to diminish the mass and slug diameter, at which it can enter the hole and increase explosion yield, especially in cases of oil rocks.

The process realising hypercumulation can be carried out in a number of ways, which can be employed in the various constructions depending on requirements. These include situations when required inorganic or organic substances, their compositions with metal, pure metals and alloys not decomposing under the impact of high pressure and vice versa turning under such pressures into a gas medium or other components - can be placed into a hole perforated in a well, cement and rock.

To ensure hypercumulative process and improving the characteristics of cumulation, special - more suitable - bodies with various systems for control of the detonation wave have been created which reduce the dimensions of perforating devices and amount of debris materials in order to minimally pollute the borehole and effectively carry out hypercumulation, both in its pure form and in combination with the elements of classic cumulation with a slug not exceeding the jet's maximal diameter.

Increasing the thickness of the cumulative jet requires increasing metal plasticity in order to achieve stretching of jet produced under the velocity gradient, as well as increasing the limit of jet's strength. 
Fig. 5.9 shows certain results of calculation experiments made in regime of pure hypercumulation using liners made of different materials - copper, iron, aluminium.

We managed to find the solution for increasing plasticity and strength limit of certain materials at cumulative flow due to creating anisotropic cumulative liners. There are very strict requirements set forth to liner's material in respect of its anisotropic properties and its homogeneity, and, in particular, to the grain size in the structure of the material, to structural homogeneity which appears when the material is rolled.

In its appearance and structure the material is already anisotropic. But the properties of thread like crystals inside the jet prevent the material from being homogeneous, since the properties of these threads produced from microcrystals of different crystallographic direction are different within a very broad scope. And, as we know, all this notwithstanding the jet consisting even of such thread-like crystals of different quality is capable of anomalous stretching which is also a reason of its expanding capacity to punch the barrier.

We managed to create a special medium for cumulative covers, which is anisotropic from the point of view of normal approach to material and ideal for being used in cumulative process. In such a material all the crystals locate in set direction - that of efficient deformation at cumulative explosion. Material with such a structure beyond the blast front attains a state close to thermodynamic balance. All the crystals stretch similarly turning into long threads. In such a medium there practically does not exist limitations for the size of grain crystals. Cumulative jet consists of long homogeneous thread-like crystals. Perforation does not depend on the size of crystals inside the liner and increases due to ever greater enlargement of a flying jet. In the experiment, even in the absence of optimal testing conditions, the effect of the charge increases. The abatement of the second viscosity of the medium characteristic for unbalanced processes at its flowing in the process of exploding can increase the mass of material turning into cumulative jet related both with the process of its formation and with decreasing energy intake for heating the material. For the first time cumulative process in the experiment was done with the liner having anomalously large crystals which in certain experiments attained the length of $1 \mathrm{~cm}$ and which do not affect the action of the product.

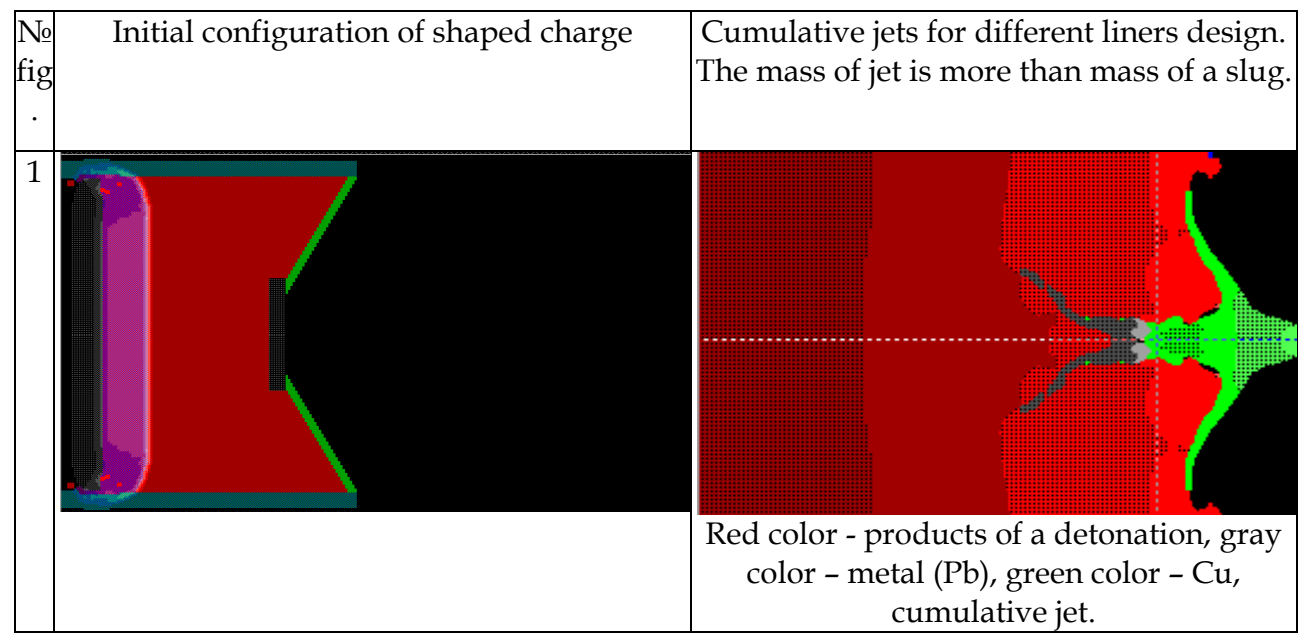




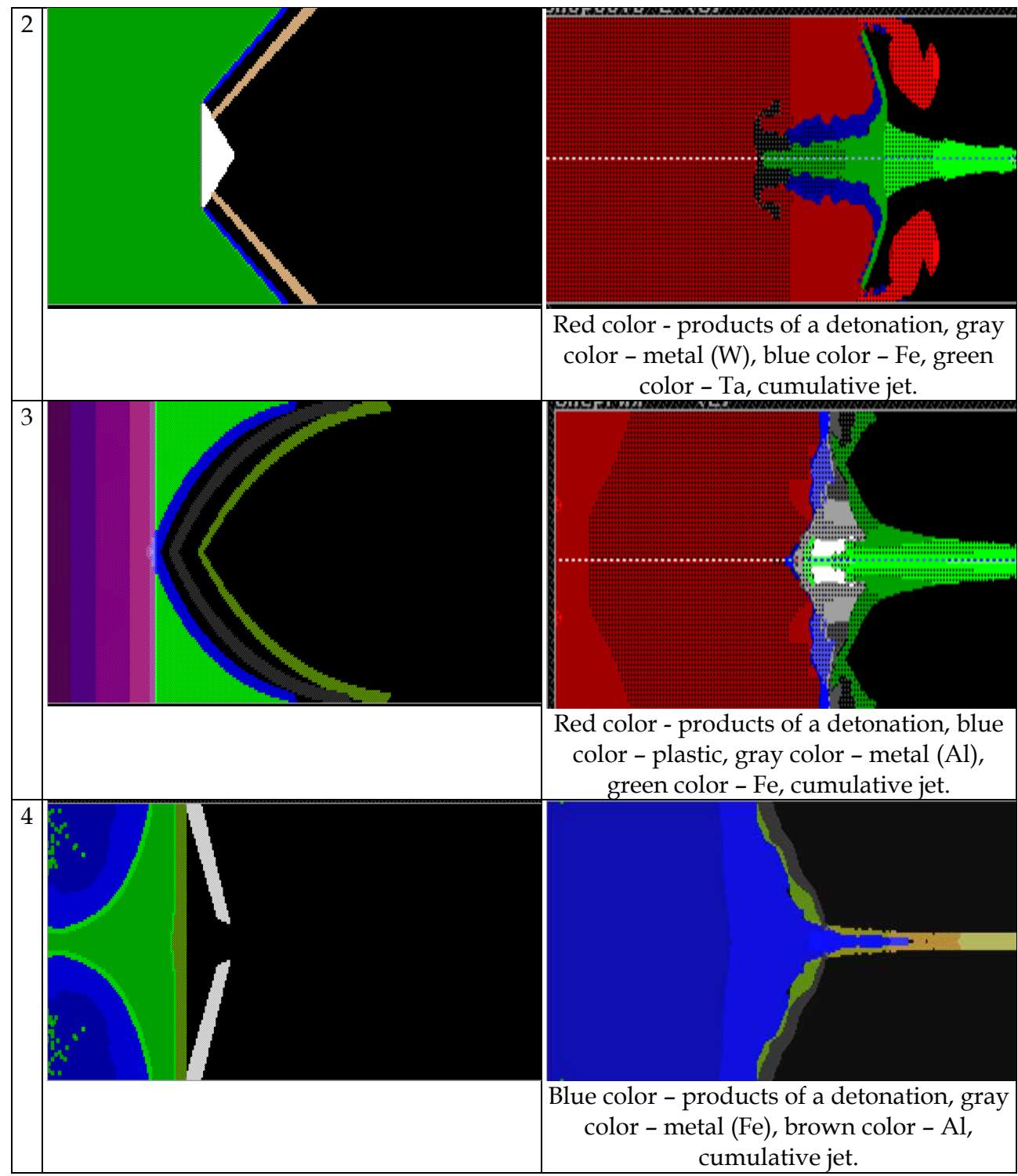

Fig. 5.9.

\section{References}

[1] Harlow, Francis H. (1964). The particle-in-cell computing method for fluid dynamics. Methods Comput. Phys. 3: 319-343.

[2] Marder B.H. (1975). GAP - a PIC-type fluid code / / Math. Of comput., v.29, N130. 
[3] Frederic A. Rasio. Particle Methods in Astrophysical Fluid Dynamics. Retrieved from http://ciera.northwestern.edu/rasio/Papers/58.pdf

[4] Minin V.F. (1984) Complex of resources of express simulation on the computer of processes of physics of explosion and flow. The paper was presented at the $3 \mathrm{All}-$ Union schools - seminars on physics of explosion and application of explosion in experiment, June 25-29, Krasnoyarsk. (in Russian)

[5] Minin V.F. (1986) Computer science and computing experiment. The paper was presented at All-Union conference "Computer science and automation of handle", Kiev, April 1-3. (in Russian)

[6] Minin V.F. et al (1989). Development and realization of the methods of numerical modeling of nonstationary flow of multicomponent compressible media by means of multiprocessor PS-2000. Preprint of the Institute of control problems. Moscow.P.1-54. (in Russian). See also: Minin V.F., Minin I.V., Minin O.V. The calculation experiment technology. - Proc. of the Int. Symp. on Intense Dynamic Loading and its Effects.- Chengdu, China, June 9-12, 1992, p.431-433.

[7] Minin V.F. et al (1989). Numerical modeling of nonregular interaction of the shock wave in condense matter. Preprint of the Institute of chemical physics. Moscow.-P.1-72. (in Russian)

[8] Minin V.F. et al (1989). Numerical simulation of nonstationary high energy processes using real equation of metals state. / Collection of works: Investigations of substance properties in extreme conditions (IHT, USSR Academy of Sciences)-P. 89-96. (in Russian)

[9] Minin V.F., Alekseev A.S., Kryukov B.P. (1989) The cosmogenios factors of effect on an earth's surface. - Scientific seminar on mathematical modelling and prediction natural and technogenios catastrophes, September 15-16, Computer centre SB AS USSR.

[10] V. F. Minin (1964), Zh. Prikl. Mekh. Tekh. Fiz., No. 3, see also: High-velocity impact phenomena, ed by R.Kinslow, Academic Press N.Y. and London, 1970. - 579 p.

[11] V.F.Minin et al (1984). Thermophysical and Gasodynamical Problems of Meteorite Defense of Cosmic Apparatus "VEGA". - Teplofiz. Vys.Temp., 22, v.5 , P.964-983.

[12] Minin V.F., Anisimov A.I., Sagdeev R.Z., Fortov V.E. (1986) Shock wave and extreme state of conditions in the Vega project of comet Galley investigation // 8 Symp. on explosion, Tashkent, 1986.

[13] Minin V.F. et al (1987). Hydrodynamic cumulative effects in conic thermonuclear targets. Book of abstracts 18-th ECLIM, Prague, May 4-8, p.187.

[14] Minin V.F. et al (1989). The numerical modeling of non-regular shock wave reflection in condencing matter. / Preprint of the Inst.of chemical physics. Moskow, p.1-71. (The paper was presented in the all-union conf. "Equation of state", February 1988).

[15] Minin V.F, Pyalling A.A., Kryukov B. P., Landin A.A., Ternovoi V.Ya., Fortov V.E. (1996) Influence of conditions of initiation on flow regimes at creation of the Mach configuration, Book of abstracts of the International conference "Mathematical models and numerical methods of a continuum mechanics ", Novosibirsk, May 27 - June 2.

[16] Minin I.V., Minin O.V. (1992) The strong shoch-wave generation by means of space modulation of laser beams interaction with target./The paper was presented in 
International Conf. "Physics and gasodynamics of shock waves", May 27-June 2, Minsk, Belarus.

[17] Minin I.V. and Minin O.V. (1990) The methods of the plasma jets control in the cone ablations. The paper was presented in: All Union Conf. "Zababachin's scientific readings", Janyary 16-19, Chelyabinsk-70; See also: I.V.Minin, O.V.Minin. Analytical and computation experiments on forced plasma jet formation. - Proc. of the Int. Symp. on Intense Dynamic Loading and Its Effects.- Chengdu, China, June 9-12, 1992, P. 588-591.

[18] V.F. Minin, I.V. Minin, O.V.Minin (2006). Criteriun of jet formation on axisymmetrical shaped charge / / Izv. VUZov ,N6 (27), pp. 380-389. (In Russian)

[19] Minin V.F., Minin I.V., Minin O.V. (1991) The dynamics of shock wave focussing with the elements of diffraction quasioptics./ Book of Abstracts the 18 Int. Symp. on Shock Waves. Sendai, July 21-26.-P.39-40.

[20] Minin I.V., Minin O.V. (1992) Diffractional quasioptic. - Moskow: InformTei, - 180 p. (in Russian). See also: Minin O.V. and Minin I.V. Diffractive optics of millimeter waves. IOP Publisher, Boston-London, 2003.

[21] I.V. Minin, O.V.Minin (2010). Explosive pulsed plasma antennas for information protection. Chapter in: "Microwave and Millimeter Wave Technologies: Semiconductor Devices, Circuits and Systems", ISBN 978-953-307-031-5, IN-TECH, Austria 2010

[22] Dushin V.R., Minin I.V., Minin O.V. et al. (1989) Investigation of the space supersonic flows of the axisymetrical body by the real gas.- Vestnik MGU, v1, \#4, 41-49. (in Russian)

[23] Minin V.F., Musatov V.V., et al. (1986) Modification of the "large particles" method for 2D non-stationary problems of continues mechanics// Mechanics of high-speed process, Novosibirsk, N73, pp. 78-85. (in Russian)

[24] V.F. Minin, I.V. Minin, O.V.Minin. (2000) The mathematical modelling of joint and magnetic-pulse welding processes / / Vestnic SF Moskow State University of press, vol 1. M., P.9-20. (in Russian)

[25] V.F. Minin, et al. (1998) Application of computational experiments in the study of the formation of the casting. Proc. of the conference "Problems of industrial crystallization and computer modeling of metallurgical technologies", June 1-3, Izhevsk, pp.88- 89. (in Russian)

[26] V.F. Minin. The interaction of underwater shock wave with a bubble curtain. PhD dissertation, Novosibirsk, 1959, Institute of hydrodymanics of SB of Russia, 117 p.

[27] Kedrinskii V.K., Soloukhin R.I. (1961) Compression of a spherical gas cavity in water shock wave. PMTF, № 1.

[28] Minin V.F., Minin I.V., Minin O.V. (2010) Method and devices of high-speed cumulative jet formation for perforation of boreholes with big diameter and without slug. Patent RU № 2009145359/20(064634) 


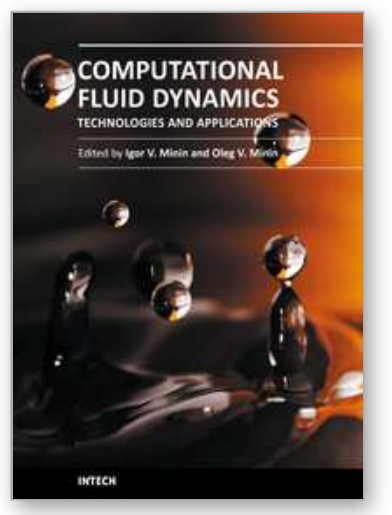

\section{Computational Fluid Dynamics Technologies and Applications}

Edited by Prof. Igor Minin

ISBN 978-953-307-169-5

Hard cover, 396 pages

Publisher InTech

Published online 05, July, 2011

Published in print edition July, 2011

This book is planned to publish with an objective to provide a state-of-art reference book in the area of computational fluid dynamics for CFD engineers, scientists, applied physicists and post-graduate students. Also the aim of the book is the continuous and timely dissemination of new and innovative CFD research and developments. This reference book is a collection of 14 chapters characterized in 4 parts: modern principles of CFD, CFD in physics, industrial and in castle. This book provides a comprehensive overview of the computational experiment technology, numerical simulation of the hydrodynamics and heat transfer processes in a two dimensional gas, application of lattice Boltzmann method in heat transfer and fluid flow, etc. Several interesting applications area are also discusses in the book like underwater vehicle propeller, the flow behavior in gas-cooled nuclear reactors, simulation odour dispersion around windbreaks and so on.

\section{How to reference}

In order to correctly reference this scholarly work, feel free to copy and paste the following:

Vladilen F. Minin, Igor V. Minin and Oleg V. Minin (2011). Calculation Experiment Technology, Computational Fluid Dynamics Technologies and Applications, Prof. Igor Minin (Ed.), ISBN: 978-953-307-169-5, InTech, Available from: http://www.intechopen.com/books/computational-fluid-dynamics-technologies-andapplications/calculation-experiment-technology

\section{INTECH}

open science | open minds

\author{
InTech Europe \\ University Campus STeP Ri \\ Slavka Krautzeka 83/A \\ 51000 Rijeka, Croatia \\ Phone: +385 (51) 770447 \\ Fax: +385 (51) 686166 \\ www.intechopen.com
}

\author{
InTech China \\ Unit 405, Office Block, Hotel Equatorial Shanghai \\ No.65, Yan An Road (West), Shanghai, 200040, China \\ 中国上海市延安西路65号上海国际贵都大饭店办公楼 405 单元 \\ Phone: +86-21-62489820 \\ Fax: $+86-21-62489821$
}


(C) 2011 The Author(s). Licensee IntechOpen. This chapter is distributed under the terms of the Creative Commons Attribution-NonCommercialShareAlike-3.0 License, which permits use, distribution and reproduction for non-commercial purposes, provided the original is properly cited and derivative works building on this content are distributed under the same license. 